\title{
Tracking quintessence by cosmic shear
}

\section{Constraints from VIRMOS-Descart and CFHTLS ${ }^{\star}$ and future prospects}

\author{
C. Schimd ${ }^{1,2}$, I. Tereno ${ }^{2,3}$, J.-P. Uzan ${ }^{2}$, Y. Mellier ${ }^{2,4}$, L. van Waerbeke ${ }^{5}$, E. Semboloni ${ }^{2}$, H. Hoekstra ${ }^{6}$, \\ L. Fu' ${ }^{2}$, and A. Riazuelo ${ }^{2}$ \\ ${ }^{1}$ DSM/DAPNIA, CEA/Saclay, 91191 Gif-sur-Yvette Cedex, France \\ e-mail: carlo.schimd@cea.fr \\ 2 Institut d'Astrophysique de Paris, UMR 7095 CNRS, Université Pierre \& Marie Curie, 98bis bd Arago, 75014 Paris, France \\ 3 Departamento de Fisica, Universidade de Lisboa, 1749-016 Lisboa, Portugal \\ 4 Observatoire de Paris - LERMA, 61 avenue de l'Observatoire, 75014 Paris, France \\ 5 Department of Physics and Astronomy, University of British Columbia, 6224 Agricultural Road, Vancouver V6T 1Z1, Canada \\ 6 Department of Physics and Astronomy, University of Victoria, Victoria V8P 52C, Canada
}

Received 7 March 2006 / Accepted 11 June 2006

\begin{abstract}
Aims. Dark energy can be investigated in two complementary ways, by considering either general parameterizations or physically well-defined models. Following the second route, we explore the observational constraints on quintessence models where the acceleration of our universe is driven by a slow-rolling scalar field. Using weak lensing data to investigate high-energy motivated models of dark energy for the first time, the analysis focuses on cosmic shear, examining how weak lensing surveys can constrain dark energy, discussing the limitations due to the lack of knowledge of the non-linear regime, and combining with type Ia supernovae data and cosmic microwave background observations to lift some degeneracies.

Methods. Using a Boltzmann code that includes quintessence models along with a weak lensing add-on code, we determine the shear power spectrum and several two-point statistics, describing the non-linear regime by two different mappings. The likelihood analysis completing the pipeline, based on a grid method, uses the "gold set" of supernovae Ia, VIRMOS-Descart and CFHTLS-deep and -wide data for weak lensing; we also explore larger angular scales, using a synthetic realization of the complete CFHTLS-wide survey, as well as of space-based mission surveys. WMAP-first year data are used for the normalization and to broadly define the location of the first acoustic peak constraining the quintessence parameter space.

Results. Two classes of cosmological parameters are discussed: $i$ ) those accounting for quintessence affect mainly geometrical factors; ii) cosmological parameters specifying the primordial universe strongly depend on the description of the non-linear regime. This dependence is confirmed using wide surveys, by discarding the smaller angular scales to reduce the dependence on the nonlinear regime. For a flat universe and a quintessence inverse-power-law potential with slope $\alpha$, the joint analysis gives $\alpha<1$ and $\Omega_{\mathrm{Q} 0}=0.75_{-0.04}^{+0.03}$ at a $95 \%$ confidence level, whereas $\alpha=2_{-2}^{+18}, \Omega_{\mathrm{Q} 0}=0.74_{-0.05}^{+0.03}$ when including supergravity corrections.
\end{abstract}

Key words. gravitational lensing - cosmology: theory - cosmological parameters - methods: data analysis

\section{Introduction}

Cosmological observations provide increasingly compelling evidence that the expansion of the universe is accelerating and that the cosmic history of the universe today seems dominated by another component than its matter and radiation content (see e.g. Peebles \& Ratra 2003; Carroll 2001; Padmanabhan 2003; Copeland et al. 2006; Peter \& Uzan 2005, Chap. 12 for reviews and references therein). If so, one of the most challenging issues of fundamental physics is to understand the cause of this acceleration, a question often referred to as the nature of the dark energy. Various solutions, from the introduction of a new type of

^ Based on observations obtained with MegaPrime/MegaCam, a joint project of CFHT and CEA/DAPNIA, at the Canada-France-Hawaii Telescope (CFHT) which is operated by the National Research Council (NRC) of Canada, the Institut National des Sciences de l'Univers of the Centre National de la Recherche Scientifique (CNRS) of France, and the University of Hawaii. This work is based in part on data products produced at TERAPIX and the Canadian Astronomy Data Centre as part of the Canada-France-Hawaii Telescope Legacy Survey, a collaborative project of $\mathrm{NRC}$ and CNRS. matter to modification of general relativity to describe the gravitation interaction, have been considered. A classification of these models with some relevant observational tests that can help to distinguish each class from their underlying new physics is discussed in Uzan (2004) and Uzan et al. (2004).

Dark energy appears in the Friedmann equations through its effective density and pressure. Data are usually interpreted assuming the validity of the Copernican principle (so that the dynamics of spacetime is completely described by a single function, the scale factor $a$ ) and the validity of Einstein equations (and thus the standard Friedmann equations), so that the density and pressure of the dark energy component are defined by $\rho_{\mathrm{de}}=(3 / 8 \pi G)\left(H^{2}+K / a^{2}\right)-\rho_{\mathrm{m}}-\rho_{\mathrm{r}}$ and $P_{\mathrm{de}}=(-1 / 8 \pi G)(\ddot{a} / a+$ $\left.H^{2}+K / a^{2}\right)$, where $H=\dot{a} / a$ is the Hubble parameter and a dot refers to a derivative with respect to the cosmic time, while $\rho_{\mathrm{m}}$ and $\rho_{\mathrm{r}}$ are the density of pressureless matter and radiation, respectively. It follows that the equation of state of the dark energy corresponds to

$3 \Omega_{\mathrm{de}} w=-1+\Omega_{K}+2 q$ 
where $q=-a \ddot{a} / \dot{a}^{2}$ is the deceleration parameter and $\Omega_{K}=$ $-K / a^{2} H^{2}$. From this point of view, $w$ characterizes the dynamics of the cosmic expansion. More precisely, it parameterizes the deviation $H(z)-\bar{H}(z)$ between the Hubble function of the observed Universe, $H(z)$, and that predicted for a universe filled only with pressureless matter and radiation, $\bar{H}(z)$. It is therefore equivalent to specifing $w(z)$ or $H(z)-\bar{H}(z)$. However, when general relativity is assumed to describe gravity, $w$ reduces to $P_{\mathrm{de}} / \rho_{\mathrm{de}}$ so that, in addition to the deviation from $\bar{H}(z)$, it also gives some insight into the properties of dark energy (see e.g. Martin et al. 2005, for a case in which $w$ does not reduce to the equation of state of a matter component).

Although an effective equation of state derived from observations is a key piece of empirical information on the rough nature of dark energy, a detailed description of its properties demands more thoughtful data interpretation. For example, all geometrical observables rely on the integration of the Hubble parameter, hence on a double integration of the equation of state $w$, that eventually dilutes or totally washes out its possible redshift dependence. If $w$ is close to -1 , as observations tend to indicate, then it is generally difficult to demonstrate by geometrical tests that $w \neq-1$ or that $\mathrm{d} w / \mathrm{d} z \neq 0$; both would exclude a pure cosmological constant. Exploring early properties of dark energy models would be even more challenging since for $w \simeq-1$ the ratio between the matter and dark energy densities scales approximatively as $(1+z)^{3}$ so that dark energy is dynamically negligible at redshift $z \gtrsim 2$. It leaves little freedom to determine the scaling of the dark energy density and to demonstrate that it is not properly described by a power law $\left[(1+z)^{n}\right]$, as would be the case for a constant $w$ (see e.g. Kujat et al. 2002).

From the theoretical point of view, two routes can be followed. One can either exhibit a general "model-independent" parameterization of the equation of state of the dark energy, as discussed in the previous paragraph, or rely on a completely specified theoretical models. A useful parameterization has to be realistic, in the sense that it should reproduce predictions of a large class of models, it has to minimize the number of free parameters, and should be simply related to the underlying physics (see e.g. Linder \& Huterer 2005). Because the result of the analysis will necessarily have some amount of parameterization dependence (Basset et al. 2002), choosing the specified physical model strategy seems preferable to breaking degeneracies. In particular, it enables one to compute their signature without any ambiguity both in low and high redshift surveys, such as the cosmic microwave background (CMB). The increasingly flourishing number of models makes it difficult to provide a comprehensive set of unambiguous predictions to constrain physical models one by one with present-day observations, but there are still several benefits to exploring dark energy this way, in particular when weak lensing surveys are used together with CMB observations. This is deeply related to the evolution of dark energy properties and the growth rate of structure with look-back time, as discussed below.

At low redshift, $w$ suffices to get observables that are all functions of $H(z)$ (see for example Peebles 1993; Peter \& Uzan 2005). This is the case for all background quantities (e.g. luminosity distance, angular distance, look-back time, etc.), as well as for the linear growth factor of density perturbations. It follows that the equation of state encodes all relevant information, provided the amplitude of the power spectrum is calibrated by adding a new parameter, $\sigma_{8}$, the variance of the density perturbation on a scale of $8 h^{-1} \mathrm{Mpc}$ (see e.g. Benabed \& Bernardeau 2001; Benabed \& van Waerbeke 2003; Doran et al. 2001a). However, as far as weak lensing is concerned, it was shown
(Benabed \& van Waerbeke 2003; Benabed \& Bernardeau 2001) that, for a fixed redshift of the sources, the modification of the growth factor in the linear regime was degenerate with the normalization factor. Hence normalizing on the CMB avoids this problem, while at the same time it is important to describe the non-linear regime. The use of CMB, together with weak lensing data, is therefore a logical way to constrain specified theoretical models, beyond description by an empirical equation of state.

At higher redshift, one would need to include a description of the evolution of the dark energy perturbations, in particular to relate the amplitude of the matter density power spectrum to the one of the primordial power spectrum. In particular, this effect becomes increasingly important as $w$ approaches zero (Benabed $\&$ Bernardeau 2001). Note that dark energy perturbations have a non-adiabatic component that also requires a detailed model to be described. This depends on the physical model of dark energy and cannot be incorporated in a simple model-independent way.

While the ability of lensing data to constrain the equation of state of dark energy has been widely studied (Benabed \& van Waerbeke 2003; Hu \& Jain 2004; Jain \& Taylor 2003), there have been very few analyses with real data. Hoekstra et al. (2006) and Semboloni et al. (2006) used the CFHTLS wide and deep data to constrain a constant equation of state. Jarvis et al. (2005) analyzed the 75 square-degree CTIO lensing survey, combined with type Ia supernovae ( $\mathrm{Sn}$ Ia) data and $\mathrm{CMB}$, assuming a constant equation of state and a parameterization of the form proposed by Chevallier \& Polarski (2001) and then by Linder (2003). In this article, we consider a class of completely defined quintessence models, realized by a selfinteracting scalar field; this extension of the standard $\Lambda \mathrm{CDM}$ model involves only one additional parameter, as needed to characterize the self-interacting potential of the quintessence field. All observational signatures (Sn Ia, lensing, CMB) can be explicitly computed, with no ambiguity in the way of dealing with the perturbations of dark energy. It follows that, as discussed above, we will be able to normalize our initial power spectrum on the CMB angular power spectrum and, as a consequence, $\sigma_{8}$ and any possible dependence on the shape of the analytical fit of the transfer function will disappear from our discussion; the value of $\sigma_{8}$ will be an output of each model. The problem of the pivot redshift (see Sect. 2.2) that appears when combining different datasets also disappears in that approach. As a conclusion, this approach is very efficient in terms of the number of extra-parameters and of the interpretation of the data analysis. Let us emphasize that, even though we also consider Sn Ia and CMB data, we focus on weak lensing - cosmic shear data. This article, being the first analysis of the CFHTLS data for dark energy studies, illustrates the power and the problems of a lensing survey for studying dark energy.

The article is organized as follows. In Sect. 2, we define the quintessence models we are considering and recall their main properties. We also compare them to various parameterizations proposed in the literature. Section 3 focuses on cosmic shear. After a review of theoretical issues, we describe the weak lensing data used for our analysis and the computational pipeline, and finally we outline the likelihood analysis of real (Sect. 3.5) and synthetic (Sect. 3.6) datasets. In Sect. 4 we combine weak lensing data with type Ia supernovae and CMB temperature anisotropies; Fig. 10 and Table 1 summarise the constraints on quintessence parameters. We finish, in Sect. 5, by an estimation of the proficiency of two possible space-based wide-field imagers to unveil the nature of dark energy. 


\section{Modelling dark energy}

Two complementary ways of accounting for the dark energy are possible, either by looking at a definite, physical (classes of) model(s) or by parameterizing its equation of state. In this section, we discuss the advantages of the former route, introducing the specific models we consider. In particular, it would be worth evaluating to what accuracy constraints of the order of $1 \%$ on a given parameterization constrain physical models.

\subsection{Quintessence models}

In this work, we consider the simplest class of quintessence models (Caldwell et al. 1998) in which a scalar field $Q$ is slowrolling in a runaway potential. Numerous forms of potentials have been proposed (see e.g. Copeland et al. 2006, for a review) but we restrict ourselves to two classes of potentials.

The first class of potentials is an inverse power law (Ratra \& Peebles 1988; Wetterich 1988)

$$
V(Q)=M^{4}\left(Q / M_{\mathrm{p}}\right)^{-\alpha},
$$

often called the Ratra-Peebles (RP) potential, and $M_{\mathrm{p}} \equiv$ $(8 \pi G)^{-1 / 2}$ is the reduced Planck mass. The potential depends on two free parameters: $\alpha$ is a positive index while $M$ is a mass scale that has to be adjusted to fit $\Omega_{\mathrm{Q} 0}$, once $\alpha$ is given. In particular, if $\Omega_{\mathrm{Q} 0}$ dominates then $M$ and $\alpha$ are related by

$\log \left(\frac{M}{1 \mathrm{GeV}}\right) \sim \frac{19 \alpha-47}{\alpha+4}$.

The second class of potential is an extension of the previous one that takes supergravity (SUGRA) corrections into account when $Q \sim M_{\mathrm{p}}$ (Brax \& Martin 1999),

$V(Q)=M^{4}\left(Q / M_{\mathrm{p}}\right)^{-\alpha} \exp \left(Q^{2} / 2 M_{\mathrm{p}}^{2}\right)$.

Both potentials have similar dynamics, as long as $Q \ll M_{\mathrm{p}}$, but differ at low redshift, in particular concerning their equation of state. In the SUGRA case, it is pushed toward -1 , so one expects $w_{0} \sim-0.82$ for $\alpha=11$ and $\Omega_{\mathrm{Q}}=0.7$ (Brax \& Martin 1999).

With such well-defined models, the dynamics of the background is completely characterized by the Klein-Gordon equation for the scalar field,

$\ddot{Q}+3 H \dot{Q}+\frac{\mathrm{d} V}{\mathrm{~d} Q}=0$,

in addition to the Friedmann equation

$\left(H^{2}+\frac{K}{a^{2}}\right)=\frac{8 \pi G}{3}\left[\rho_{\mathrm{m}}+\rho_{\mathrm{r}}+\frac{\dot{Q}^{2}}{2}+V(Q)\right]$,

allowing for this new matter contribution. These equations characterize background and low redshift observations and, in particular, the linear growth factor. Let us stress that, at this level of description, one can describe the quintessence component as a fluid with a time-dependent equation of state, because the speed of sound $c_{\mathrm{s}}$ is given by

$c_{\mathrm{s}}^{2}=1+\frac{4}{3} \frac{1}{H \dot{Q}} \frac{\mathrm{d} V}{\mathrm{~d} Q}$

and because the equation of state evolves as

$\dot{w}=-3 H(1+w)\left(c_{\mathrm{s}}^{2}-w\right)$.

These models share the interesting property of possessing scaling solutions that are attractors to the dynamical evolution.

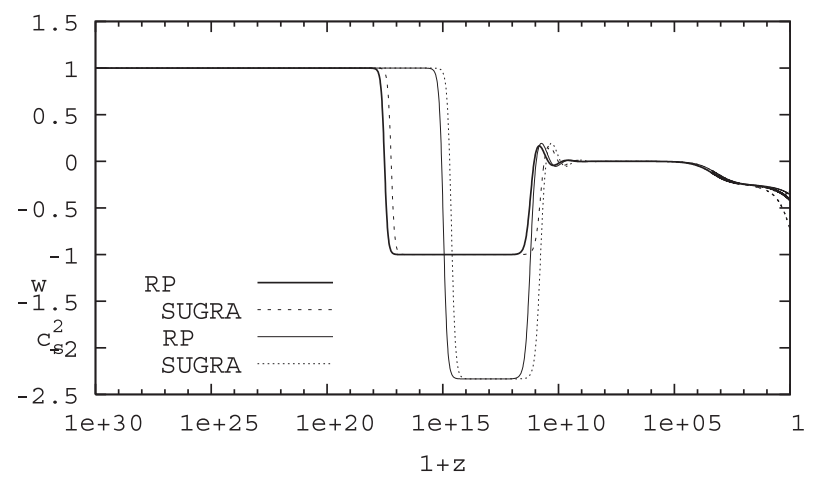

Fig. 1. Evolution of the equation of state $w$ and of the sound speed $c_{\mathrm{s}}$ with redshift $z$ for an inverse power law quintessence model with $\alpha=6$ and $\Omega_{\mathrm{Q} 0}=0.7$, including the supergravity correction or not. We recover, from high to low redshift, the kinetic $(w=1)$, slow-rolling $(w=-1)$, and tracking phases $\left(z \lesssim 10^{8}\right)$ described in the text.

Depending on the initial conditions, the dynamics starts with an early kinetic phase $\left(\dot{Q}^{2} \gg V\right)$ in which $w \sim 1$, so that $\rho_{\mathrm{Q}} \propto(1+z)^{6}$. The field behaves as $Q=Q_{i}-A(1+z)$ and it freezes to a constant value. Since the kinetic energy decreases while the potential remains constant, this regime cannot last forever. When the potential starts dominating, the equation of state shifts suddenly to $w \sim-1$. During this transition regime, $\rho_{\mathrm{Q}} \propto(1+z)^{0}$. Then, there is a potential regime that lasts until the tracking regime (Zlatev et al. 1999), during which

$w=c_{\mathrm{s}}^{2}=\frac{\alpha w_{\mathrm{B}}-2}{\alpha+2}$

where $w_{\mathrm{B}}$ stands for the equation of state of the fluid dominating the background. At that stage, the scalar field is slow-rolling so that $w<0$. Figure 1 depicts the evolution of $w$ and $c_{\mathrm{s}}^{2}$ during these various regimes.

When cosmic microwave background anisotropies and largescale structures are considered, one needs to include the description of the evolution of the perturbations and, in particular, include those of the scalar field described by

$\delta \ddot{Q}+3 H \delta \dot{Q}+\left(\frac{k^{2}}{a^{2}}+\frac{\mathrm{d}^{2} V}{\mathrm{~d} Q^{2}}\right) \delta Q+\mathcal{S}=0$

where $\mathcal{S}$ encodes the perturbations of the metric of the spacetime and $k$ is the comoving wavenumber of the perturbation. It has been shown (Brax et al. 2000; Riazuelo \& Uzan 2002) that an attraction mechanism exists for super-Hubble wavelength so that the spectrum is insensitive to the initial conditions for the scalar field.

\subsection{Parameterizing the equation of state}

Most data, and especially supernovae data, are being analyzed using a general parameterization of the equation of state. These parameterizations are useful for extracting model-independent information from the observations, but the interpretation of these parameters is not always straightforward. In this paragraph, we recall the properties of some interesting parameterizations and compare them to the quintessence models we are considering.

Recently, weak lensing data (Semboloni et al. 2006; Hoekstra et al. 2006), as well as Sn Ia (Riess et al. 2004; Astier et al. 2006), baryon acoustic oscillations (Eisenstein et al. 2005), and CMB (Spergel et al. 2006) data, were used to put constraints on $w$ under the assumption it is a constant parameter. In that 
particular case, one has two characteristic redshifts, $z_{a}$ and $z_{\mathrm{de}}$, defining the beginning of the acceleration phase $\left[\ddot{a}\left(z_{a}\right)=0\right]$ and of the domination of the dark energy $\left[\Omega_{\mathrm{m}}\left(z_{\mathrm{de}}\right)=\Omega_{\mathrm{de}}\left(z_{\mathrm{de}}\right)\right]$. They are given by

$\left(1+z_{a}\right)^{3 w}=-\frac{1}{1+3 w} \frac{\Omega_{\mathrm{de} 0}}{\Omega_{\mathrm{m} 0}}, \quad\left(1+z_{\mathrm{de}}\right)^{3 w}=\frac{\Omega_{\mathrm{de} 0}}{\Omega_{\mathrm{m} 0}}$

so that $z_{a}>z_{\mathrm{de}}$. When $w$ becomes negative and high in absolute value, $z_{a}$ and $z_{\mathrm{de}}$ tend to zero so that dark energy only just now begins to dominate the universe and is redshifted in a way that it does not affect even the low-redshift observables. It follows that we expect the data to be insensitive to the value of $w$ in that regime so that one can get only an upper limit on its value, as found by Semboloni et al. (2006) and Hoekstra et al. (2005). Indeed, such a situation cannot be achieved with a physical model as considered here, because by construction it imposes that $w \geq-1$. It follows that our approach is a physically motivated way of imposing a prior on $w$. Note also that Fig. 1 shows that $w=$ constant is not a good approximation of these two classes of models. At best the constraint on a constant $w$ can be related to some redshift average of the equation of state. For these reasons, it is difficult to deduce a constraint on the physical models from a constraint on a constant $w$, even though the results of Table 1 are compatible with them.

Beyond a constant equation of state, general parameterizations of the equation of state as

$w(a)=w\left(a_{0}\right)+\left[w\left(a_{\mathrm{m}}\right)-w\left(a_{0}\right)\right] \Gamma\left(a, a_{\mathrm{t}}, \Delta\right)$

were shown to allow an adequate treatment of a large class of quintessence models (Corasaniti \& Copeland 2003; Basset et al. 2002). Such a parameterization involves four parameters $\left\{w\left(a_{0}\right), w\left(a_{\mathrm{m}}\right), a_{\mathrm{t}}, \Delta\right\}$ and a free function $\Gamma$ varying smoothly between one at high redshift to zero at $z=0$. Even though it reproduces the equation of state of most quintessence models, it is not economical in terms of the number of parameters since most quintessence potentials involve one or two free parameters. If one assumes that the parameterization is supposed to describe the dynamics of a minimally coupled scalar field, the knowledge of $w$ is sufficient, but one would need more information in a more general case. The background dynamics depends on the potential and its first derivative, which can be related to $w$ and $\dot{w}$. Accounting for perturbations, one needs to know the second derivative of the potential (see Eq. (10)), which can be inferred from $\ddot{w}$ (Dave et al. 2002).

Since we expect dark energy to have observable consequences on the dynamics only at late time, one can consider an equation of state obtained as a Taylor expansion around a pivot point $a_{\text {pivot }}$,

$w(a)=w_{\text {pivot }}+w_{a}\left(a_{\text {pivot }}-a\right)$.

This form, which depends on only two parameters $\left\{w_{\text {pivot }}, w_{a}\right\}$, is a generalization of the parameterization proposed by Chevallier $\&$ Polarski (2001) and then Linder (2003), where $a_{\text {pivot }}=1$. The value of $a_{\text {pivot }}$ is chosen in such a way that the errors in $w_{\text {pivot }}$ and $w_{a}$ are uncorrelated (Hu \& Jain 2004). In particular, it was argued that it is important to choose $a_{\text {pivot }} \neq 1$ for distancebased measurements. Two considerations are in order when using such a parameterization. First, the redshift range for which this is a good approximation of the equation of state is unknown. Clearly, compared with the form inferred by the scalar field dynamics, it is unlikely to describe dark energy up to recombination time; see Fig. 2. In this respect, dark energy models defined by a Lagrangian are more suitable, yielding a definite equation

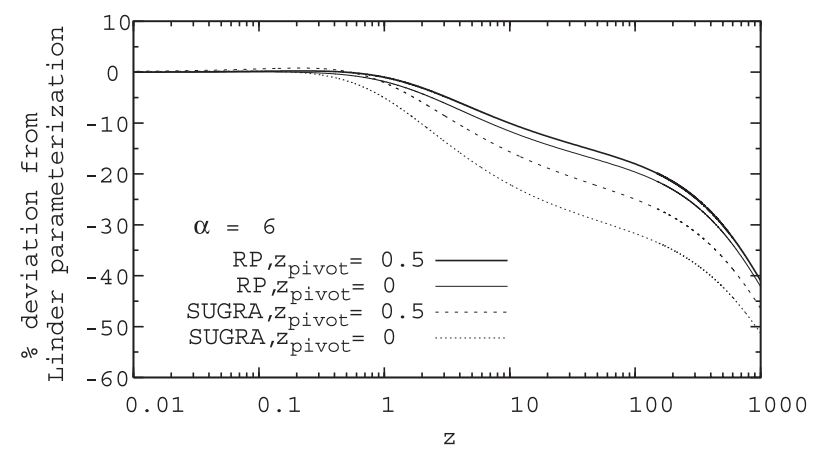

Fig. 2. Deviation of the quintessence equation of state for Ratra-Peebles (solid) and SUGRA (dotted) models with $\alpha=6$ from their fit using the generalized parameterization, Eq. (13), where $z_{\text {pivot }}=0$ (thick) or $z_{\text {pivot }}=0.5$ (thin). Fitting up to $z \lesssim 0.3$, a deviation larger than $2 \%$ occurs at $z \simeq 1$ for Ratra-Peebles models while at $z \simeq 0.5$ for SUGRA models.

of state as a function of redshift, hence more general than a Taylor expansion around a pivot point. Eventually, one can read out the values of $w$ and $w_{a}$ at whatever redshift and check the consistency of the constraints on $\left(w_{\text {pivot }}, w_{a}\right)$ with the underlying (quintessence) model; for instance, Fig. 3 depicts the value of $w$ and $w_{a}$ at $z=0$ and $z=0.5$ for the quintessence models we use, Eqs. (2) and (4). Secondly, the pivot redshift is specific to the observable. Therefore, a complication arises when combining observables at different redshifts, such as weak lensing, $\mathrm{Sn} \mathrm{Ia}$, and CMB, generally requiring different $z_{\text {pivot }}$; it will be more difficult to infer constraints on the physical models from the constraints on the parameterization.

There is an alternative way to get a first hint to the nature of dark energy. It may be useful to consider the plane $\left(w, w^{\prime}\right)$ where $w^{\prime} \equiv \mathrm{d} w / \mathrm{d} \ln a$ is the derivative of $w$ with respect to the number of $e$-folds. It was recently shown by Caldwell $\&$ Linder (2005) and Scherrer (2005) that quintessence models occupy a narrow part of this plane. This can be understood from Eq. (8) which implies that $w^{\prime}+3\left(1-w^{2}\right)=3(1+w)\left(1-c_{\mathrm{s}}^{2}\right)$. For quintessence models, $1+w>0$, and Eq. (7) implies that $c_{\mathrm{s}}^{2}<1$ (because $\dot{Q}>0$ and $V^{\prime}<0$ ) so that

$w^{\prime}>-3\left(1-w^{2}\right)$

without any assumptions on the dynamics of the scalar field. In Caldwell \& Linder (2005), two classes of quintessence models were exhibited, namely "thawing" models, in which $w \sim-1$ initially and increases as $Q$ rolls down the potential, and "freezing" models, in which $w>-1$ initially and tends toward -1 as $Q$ rolls down the potential. "Freezing" models contain tracking models and, in particular, the Ratra-Peebles models, Eq. (2), and SUGRA models, Eq. (4), considered in this work. Using a combination of numerical simulations and physical arguments, they conclude that

$3 w(1+w)<w^{\prime}<0.2 w(1+w)$

for "freezing" models. From an observational point of view, the analysis of supernovae data (Riess et al. 2004) showed that, if the universe is flat, then $w_{0}=-1.31_{-0.28}^{+0.22}$ and $w_{0}^{\prime}=-1.48_{-0.81}^{+0.90}$ after marginalizing on $\Omega_{\mathrm{m} 0}$. If one further imposes that $w_{0}>-1$, then $w_{0}<-0.76$ and $w_{0}^{\prime}=-0.6 \pm 0.5$. In the case where $w$ is assumed constant, then $w=-1.02_{-0.19}^{+0.13}$ and $w<-0.72$ at $68 \%$ and $99 \%$ confidence levels, respectively.

This phase-space analysis creates a link between the various parameterizations and the physical models. It can be 

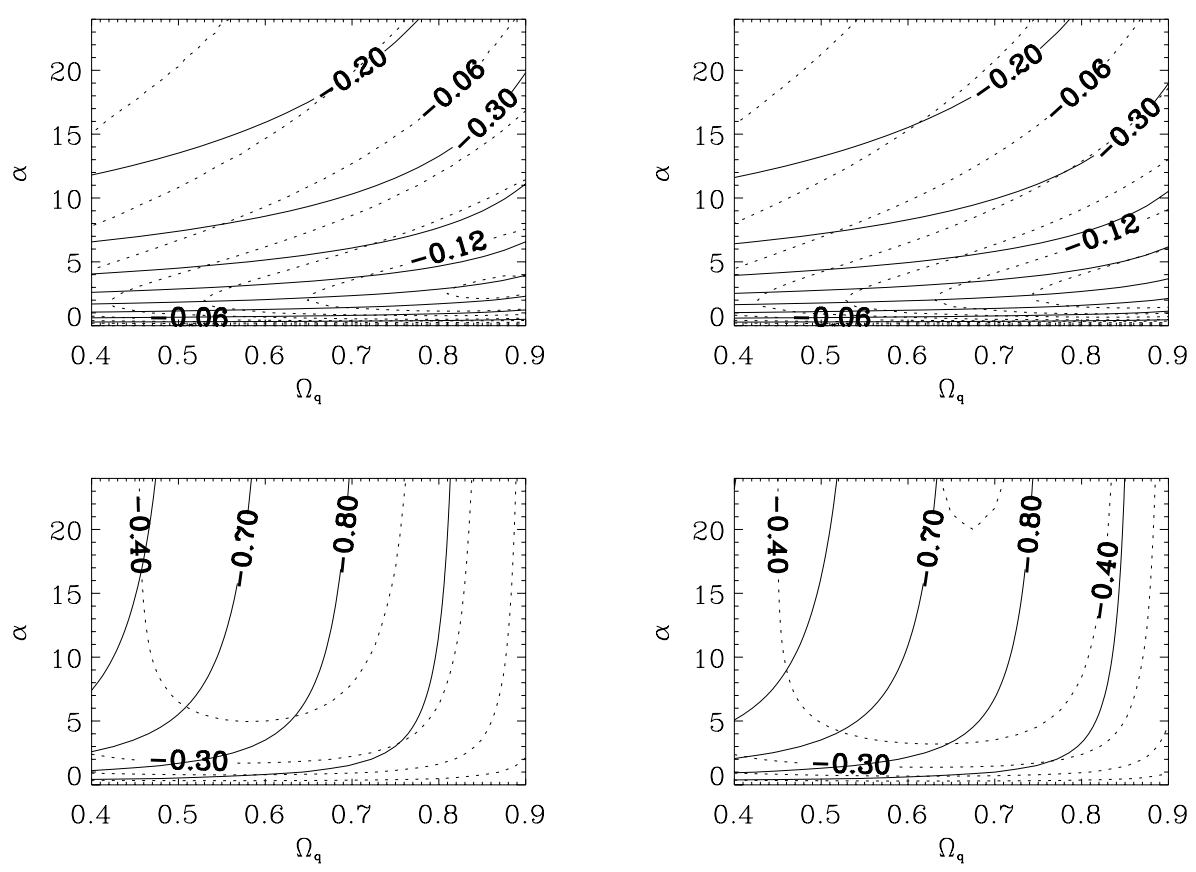

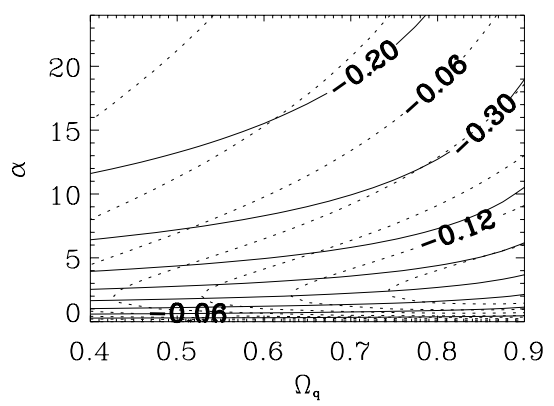

Fig. 3. Contour plots of the quintessence equation of state $w$ (solid) and its time variation $w_{a}=\mathrm{d} w / \mathrm{d} a$ (dotted) for Ratra-Peebles models (Eq. (2); upper panels) and SUGRA models (Eq. (4); lower panels), evaluated at $z=0$ (left panels) and $z=0.5$ (right panels). shown (Scherrer 2005) that different classes of models (e.g. k-essence, Chaplygin gaz, quintessence, etc.) lie in different parts, hence offering a way to distinguish between these models without measuring $w(z)$. In particular, Fig. 4 depicts the dynamics of some Ratra-Peebles and SUGRA models in the $\left(w, w^{\prime}\right)$ plane, superposed on the regions where the inequalities (14) and (15) hold. Notice that the trajectories for the SUGRA potential are essentially the same for the Ratra-Peebles one, deviating just at low redshift towards the cosmological constant solution.

\subsection{Summary}

Quintessence models require only two parameters to describe the whole dynamics (with no redshift limitation). Compared to a pure cosmological constant, described by only one number, this gives us one extra parameter. In terms of extra-parameter with respect to a standard $\Lambda \mathrm{CDM}$, this is equivalent to considering a constant equation of state for dark energy.

On the other hand, a parameterization of the equation of state of the dark energy is sufficient for describing the low redshift universe. But, the parameterizations that describe quintessence models accurately involve at least four extra-parameters. It is thus more economical to work with the physical model directly. In that case, the evolution of perturbations can be inferred from $w$, but this is not the case in more general situations.

Parameterizations with fewer parameters have a smaller dynamical range and are likely to be bad descriptions of dark energy at high redshift, notably for CMB computation. Besides, when combining datasets, the choice of the pivot redshift may induce some difficulty in interpreting the constraints on the parameters in terms of constraints on the physical models. Considering a well-defined physical model directly instead of a parameterization is thus economical for the number of extra parameters and avoids the problem of the pivot redshift. It allows us to compute the prediction of the models at all redshifts ( $\mathrm{Sn} \mathrm{Ia,} \mathrm{weak} \mathrm{lensing,}$ and CMB). On the other hand, it only concerns a small class of models.

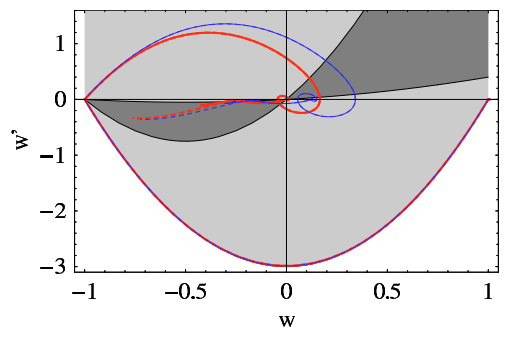

Fig. 4. Dynamics of the two quintessence models in the plane $\left(w, w^{\prime}\right)$. The shaded regions correspond to the constraints (14) in light gray and (15) in dark grey. We have considered a Ratra-Peebles (solid) and SUGRA (dash) models with $\alpha=6$ (thick/red) and $\alpha=11$ (thin/blue).

\section{Cosmic shear}

Gravitational lensing by large-scale structures of the universe produces weak distortion fields and collectively modify the shape of background galaxies (see e.g. Bartelmann \& Schneider 2001; Mellier 1999; Réfrégier 2003). Though this is a very weak signal and a challenging task, it has been detected almost simultaneously by van Waerbeke et al. (2001), Wittman et al. (2000), Kaiser et al. (2000), Bacon et al. (2000), and it is now routinely observed by many groups around the world (see van Waerbeke \& Mellier 2003; Hoekstra 2003 for recent reviews about observations). Over the past years the huge efforts carried out by these groups to deal with critical systematics considerably improved the reliability of the lensing signal and strengthened the ability of cosmic shear to constrain cosmological models from the statistical analyses of galaxy ellipticities.

\subsection{Generalities}

The gravitational lensing effect depends on the second-order derivatives of the gravitational potential. The convergence, $\kappa$, and the shear, $\gamma=\left(\gamma_{1}, \gamma_{2}\right)$, describe the distortion of background images by the matter along the line of sight. These components are related by

$\Delta \kappa=\left(\partial_{1}^{2}-\partial_{2}^{2}\right) \gamma_{1}+2 \partial_{1} \partial_{2} \gamma_{2}$ 
The evolution of the convergence is dictated by the Sachs equation (Sachs 1962; see Uzan \& Bernardeau 2000 for a more modern description). The convergence in the direction $\boldsymbol{\theta}$ can be related to the matter distribution integrated along the line of sight

$$
\begin{aligned}
\kappa(\boldsymbol{\theta}, \chi)= & \frac{3}{2} \frac{\Omega_{\mathrm{m} 0} H_{0}^{2}}{c^{2}} \int_{0}^{\chi} \frac{S_{K}\left(\chi-\chi^{\prime}\right) S_{K}\left(\chi^{\prime}\right)}{S_{K}(\chi)} \\
& \times \frac{\delta_{\mathrm{m}}\left[S_{K}\left(\chi^{\prime}\right) \boldsymbol{\theta}, \chi^{\prime}\right]}{a\left(\chi^{\prime}\right)} \mathrm{d} \chi^{\prime}
\end{aligned}
$$

for sources located at a radial distance $\chi$ defined by

$\chi(z)=\int_{0}^{z} \frac{\mathrm{d} z^{\prime}}{H\left(z^{\prime}\right)}$

and $S_{K}$ is the angular diameter distance given by

$$
S_{K}(\chi)=\left\{\begin{array}{ll}
\sin (\sqrt{K} \chi) / \sqrt{K} & K>0 \\
\chi & K=0 . \\
\sinh (\sqrt{-K} \chi) / \sqrt{-K} & K<0
\end{array} .\right.
$$

If the sources have a distribution given by $n_{\chi}(\chi) \mathrm{d} \chi=n(z) \mathrm{d} z$, then the effective convergence takes the form

$$
\kappa(\boldsymbol{\theta})=\int_{0}^{\chi_{\mathrm{H}}} n_{\chi}(\chi) \kappa(\boldsymbol{\theta}, \chi) \mathrm{d} \chi
$$

where $\chi_{\mathrm{H}}$ is the comoving radial distance of the horizon. Decomposing the convergence in 2-dimensional Fourier modes,

$\kappa(\boldsymbol{\theta})=\int \frac{\mathrm{d}^{2} \boldsymbol{\ell}}{2 \pi} \hat{\kappa}(\boldsymbol{\ell}) \mathrm{e}^{\mathrm{i} \boldsymbol{\ell} \cdot \boldsymbol{\theta}}$,

the shear power spectrum, defined by $\left\langle\hat{\kappa}(\boldsymbol{\ell}) \hat{\kappa}\left(\boldsymbol{\ell}^{\prime}\right)\right\rangle=P_{\kappa}(\ell) \delta^{(2)}(\boldsymbol{\ell}+$ $\left.\boldsymbol{\ell}^{\prime}\right)$, can be related to the 3 -dimensional power spectrum of matter density perturbations $P_{\mathrm{m}}$ by

$P_{K}(\ell)=\frac{9}{4} \frac{H_{0}^{4} \Omega_{\mathrm{m} 0}^{2}}{c^{4}} \int_{0}^{\chi_{\mathrm{H}}}\left[\frac{g(\chi)}{a(\chi)}\right]^{2} P_{\mathrm{m}}\left[\frac{\ell}{S_{K}(\chi)}, \chi\right] \mathrm{d} \chi$

in the small angle approximation (see e.g. Bartelmann \& Schneider 2001; Peter \& Uzan 2005, Chap. 7). Function $g$ is given by

$g(\chi)=\int_{\chi}^{\chi_{\mathrm{H}}} n_{\chi}\left(\chi^{\prime}\right) \frac{S_{K}\left(\chi^{\prime}-\chi\right)}{S_{K}\left(\chi^{\prime}\right)} \mathrm{d} \chi^{\prime}$.

Note that the window function $W(z) \equiv[g(\chi) / a(\chi)]^{2}$ peaks around $z \simeq z^{*} / 2$ for a distribution of sources $n(z)$ approximately peaked at redshift $z^{*}$. This will be useful in the choice of the pivot redshift. Let us stress that expressions (17) and (22) assume the validity of the Poisson equation and thus of general relativity. These expressions may be slightly different in more general contexts and even be used to test general relativity (see Uzan \& Bernardeau 2001; Schimd et al. 2005).

Neither $\kappa$ nor $P_{\kappa}$ are directly observable, but only filtered quantities can be obtained. Cosmic shear can be measured by various types of 2-point statistics that differ only by the chosen filtering scheme. This implies that their sensitivity to the power spectrum, and also to systematics, are different. In this work we consider two of these statistics, namely the aperture map variance defined by

$$
\left\langle M_{\mathrm{ap}}^{2}\right\rangle\left(\theta_{\mathrm{c}}\right)=\frac{288}{\pi} \int \ell P_{K}(\ell)\left[\frac{J_{4}\left(\ell \theta_{\mathrm{c}}\right)}{\ell^{2} \theta_{\mathrm{c}}^{2}}\right]^{2} \mathrm{~d} \ell,
$$

which is a bandpass estimate of the convergence power spectrum, and the top-hat shear variance

$\left\langle\gamma^{2}\right\rangle\left(\theta_{\mathrm{c}}\right)=\frac{8}{\pi} \int \ell P_{\kappa}(\ell)\left[\frac{J_{1}\left(\ell \theta_{\mathrm{c}}\right)}{\ell \theta_{\mathrm{c}}}\right]^{2} \mathrm{~d} \ell$,

which is a lowpass estimate of $P_{\kappa}$. Here $J_{n}$ are the Bessel functions of the first kind. Both statistics can be deduced from two linear combinations of the radial and tangential components of the shear variance, $\xi_{ \pm} \equiv\left\langle\gamma_{\mathrm{t}}^{2}\right\rangle \pm\left\langle\gamma_{\mathrm{r}}^{2}\right\rangle$ (see e.g. Bartelmann \& Schneider 2001), which are directly estimated from the shapes of background galaxies.

\subsection{Matter power spectrum and non-linear regime}

In the previous expressions and, in particular, in Eqs. (17) and (22), $P_{\mathrm{m}}$ refers to the full 3-dimensional power spectrum of pressureless matter, including cold dark matter and baryons.

In the linear regime, the growth factor $D_{+}$is the growing solution of

$\ddot{D}_{+}+2 H \dot{D}_{+}-\frac{3}{2} H^{2} \Omega_{\mathrm{m}} D_{+}=0$.

The second term that describes the damping due to the cosmological expansion contains all the effect of dark energy on $D_{+}$. In this regime, the total effect on lensing, Eq. (22), is similar to the one obtained from a single redshift plane (see Benabed \& van Waerbeke 2003; Schimd et al. 2005) so that the integrated growth effect is degenerated with the normalization of the spectrum.

For low-redshift sources, the lensing predictions and analysis involve the non-linear power spectrum. This regime cannot be described analytically with a perturbation approach (see however the proposal by Crocce \& Scoccimarro 2005), so one would need to rely on $N$-body simulations; see White \& Vale (2004) for a first attempt to study weak lensing by $N$-body simulations, including an effective quintessence component with a constant equation of state.

Some $N$-body simulations that include a dynamical quintessence were recently performed using the GADGET code (Dolag et al. 2003) or an adaptive refinement tree code (Klypin et al. 2003). It was argued by Dolag et al. (2003) that the halo concentration distribution around the mean value does not depend on the cosmology, while the concentration parameter depends on the dark energy equation of state at the cluster formation redshift through the linear growth factor. However, a systematic study confirming this claim is lacking. Klypin et al. (2003) and Mainini et al. (2003) show that dark energy changes the virial density contrast, $\Delta_{c}$, which induces a change in the power spectrum on small scales $\left(k \gtrsim 1 h \mathrm{Mpc}^{-1}\right)$ but, for constant $w$, the error is smaller than the error in the expected nonlinear model.

Instead of specific $N$-body simulations accomodating quintessence, in order to compute the non-linear matter power spectrum one can deal with linear-to-non-linear mappings, for instance based on the stable clustering ansatz (Hamilton et al. 1991; Peacock \& Dodds 1996) or to a halo model (e.g. Ma \& Fry 2000; Seljak 2000; Smith et al. 2003). These mappings have been tested for several cosmologies including $\Lambda$ CDM but not for dynamical dark energy models. Given their robustness and the precision level we can actually reach, we can hope that they remain valid for the class of models we are considering. Indeed this is a very strong assumption that can be justified by the fact that we do not expect the scalar field to cluster on small 
scales, so that it is unlikely to affect the small scale behaviour of matter except by its influence on the expansion rate. Arguing that the clustering scale of the quintessence field is given by its Compton wavelength, Ma et al. (1999) propose an analytic approximation for the Peacock \& Dodds (1996) formula to include quintessence with constant equation of state, claiming a $10 \%$ level of accuracy. McDonald et al. (2005) propose a recipe for extending the aforementioned mappings to $w \neq-1$ cases, attaining a better accuracy for $k \lesssim 10 \mathrm{Mpc}^{-1}$ by systematically exploring a wider parameter space. Nevertheless, upcoming weak lensing measurements require an improved description of the smaller scales physics (Huterer \& Takada 2005), eventually including hydrodynamics (White 2004; Zhan \& Knox 2004; Jing et al. 2005).

In conclusion, it seems early to decide how dark energy, and quintessence in particular, modify the mapping calibrated on $\Lambda \mathrm{CDM}$. For that reason, in this work we consider two linearto-non-linear mappings, by Peacock \& Dodds (1996) and Smith et al. (2003), and try to identify the parameters that are not sensitive to this choice. We will also try to quantify how the other parameters are affected so that we can estimate how our ignorance of the non-linear regime limits the use of weak lensing.

Hopefully, as we also show, weak lensing data in the linear regime will be able to be used. In that case, we can get an interesting constraint on the growth factor without messing with non-linear physics.

\subsection{Lensing data}

We use three sets of data for weak lensing: the VIRMOS-Descart (van Waerbeke et al. 2005), the deep field of the CFHTLS survey (Semboloni et al. 2006), and the wide field of CFHTLS (Hoekstra et al. 2006).

The details regarding the survey properties, image, and catalogue processing of VIRMOS-Descart data are described in van Waerbeke et al. (2001) and McCracken et al. (2003). The shear measurement and error analysis are described in van Waerbeke et al. (2005) and summarized in Table 2 of that paper. This survey covers an effective (the unmasked area) area of $8.5 \mathrm{deg}^{2}$ spread over four fields. It probes lensed galaxies down to the limiting magnitude $I_{A B}=24.5$, has an effective galaxy number density (after all selection processes) of $15 \mathrm{gal} / \mathrm{arcmin}^{2}$, and explores angular scales up to 50 arcmin.

For the CFHTLS deep and wide surveys, all relevant details are given in Semboloni et al. (2006) and Hoekstra et al. (2006), respectively. The deep survey covers an effective area of $2.2 \mathrm{deg}^{2}$ in three fields, down to $I_{A B}=26$, but only samples angular scales up to 30 arcmin. The effective galaxy number density is $22 \mathrm{gal} / \mathrm{arcmin}^{2}$. In contrast, the wide survey has a much larger effective area than the deep $\left(22 \mathrm{deg}^{2}\right)$ but only spread over two fields. It explores angular scales up to one degree at about the same depth as VIRMOS-Descart. The effective galaxy number density is $13 \mathrm{gal} / \mathrm{arcmin}^{2}$ (the final selection produced a catalogue somewhat less deep than VIRMOS-Descart). Note that, once completed, the wide survey will be composed of three compact areas that will sample angular scales up to 5 degrees in three independent fields so that linear scales will be explored with much more accuracy than present-day CFHTLS wide data.

Each survey used complementary photometric or spectroscopic galaxy samples to derive the redshift distribution of the lensed galaxy samples. As discussed in the VIRMOS-Descart and CFHTLS cosmic shear papers, it is convenient to describe the redshift distribution by a three-parameter function

$$
n(z)=\frac{\beta}{z_{\mathrm{s}} \Gamma\left(\frac{1+\alpha}{\beta}\right)}\left(\frac{z}{z_{\mathrm{s}}}\right)^{\alpha} \exp \left[-\left(\frac{z}{z_{\mathrm{s}}}\right)^{\beta}\right],
$$

where $\alpha$ and $\beta$ are obtained from a fit of photometric redshift distributions derived from external redshift calibration surveys. The CFHTLS and VIRMOS are different lensing data sets, but they were obtained with the same telescope (although with different instruments) and with the same exposure time. Therefore the two lensing surveys have to be calibrated using the same external redshift data set in order to preserve the homogeneity of the analysis. In van Waerbeke et al. (2005), VIRMOS was calibrated using the Hubble Deep Fields (HDF) and MS1008 (see van Waerbeke et al. 2001, for the details), while the CFHTLS lensing data (Hoekstra et al. 2006; Semboloni et al. 2006) were calibrated using only the HDF. The HDF and MS1008 data in visible and near infrared bands produced accurate photometric redshifts (Yahata et al. 2000; Athreya et al. 2002). We decided to use the HDF only for both VIRMOS and CFHTLS and abandon the MS1008 field. We have checked that the results discussed in this paper do not depend on whether or not we include the MS1008 calibration field, since the sample variance due to the use of combined redshift calibration sets is absorbed in the redshift error (van Waerbeke et al. 2006).

In the following, we use for the CFHTLS-deep survey (Semboloni et al. 2006)

$\alpha=1.9833, \quad \beta=0.6651, \quad z_{\mathrm{s}}=0.0981_{-0.0114-0.0161}^{+0.0129+0.0209}$

giving a mean redshift $\langle z\rangle=1.01$, while for the CFHTLS-wide and the VIRMOS-Descart surveys (Hoekstra et al. 2006)

$\alpha=1.35, \quad \beta=1.654, \quad z_{\mathrm{s}}=0.668_{-0.036-0.055}^{+0.035+0.053}$

giving a mean redshift $\langle z\rangle=0.76$. We quote the $1 \sigma$ and $2 \sigma$ errors. We use the same parameters for the VIRMOS-Descart and CFHTLS-wide redshift distributions, since both surveys have a similar depth and the same effective galaxy number density.

\subsection{Description of the pipeline and choice of the free parameters}

The pipeline we set up is summarized in Fig. 5. The first unit is a Boltzmann code (Riazuelo \& Uzan 2002), which solves the Einstein and Klein-Gordon equations to compute the evolution of the background and linear power spectra of perturbations in metric, matter, radiation, and quintessence fields, eventually supplying every background quantity (and notably the luminosity distance) as a function of redshift as well as CMB spectra. Notice that this code deals with several gauge choices and can also account for scalar-tensor theories of gravity, hence possibly allowing us to study deviations from general relativity in this framework, as well as extended quintessence scenarios (Uzan 1999; Amendola 2000; Chiba 1999).

Using this code we compute the CMB temperature (TT) angular power spectrum $\left(C_{\ell}\right)$ in order to fix the amplitude of the initial matter power spectrum at the redshift of the last scattering. We do it by matching the computed $C_{\ell}$ with WMAP-1yr data (Hinshaw et al. 2003) at a high multipole, to be preferred when studying quintessence since at low multipoles the integrated Sachs-Wolfe effect is dominant so that temperature anisotropies are not directly related to the density perturbations. Definitely, we use the multipole $\ell \simeq 110$ of WMAP-1yr data, where the total 


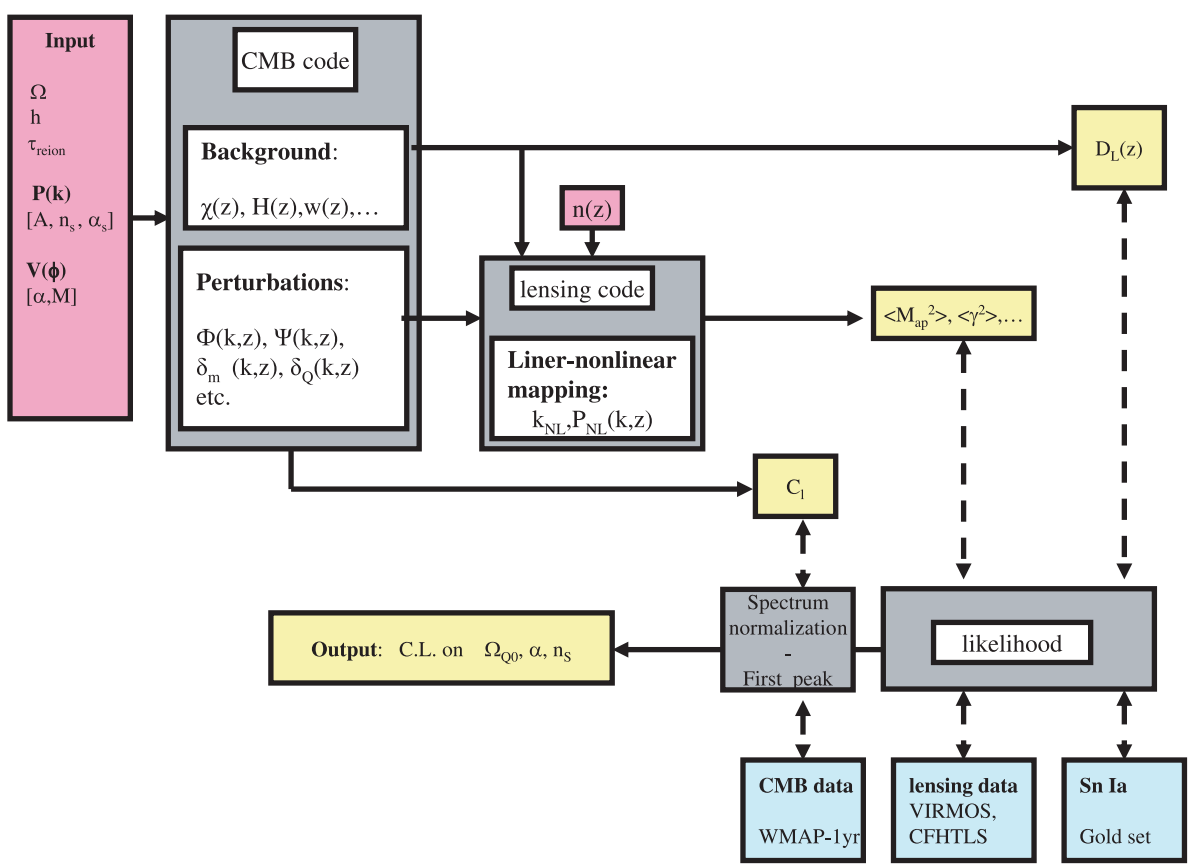

Fig. 5. Simulation-analysis pipeline, including a Boltzmann code, a lensing add-on code, and a series of codes for the grid-based likelihood analysis. See Sect. 3.4 for details. relative error of the TT spectrum is smaller than $3 \%^{1}$. This normalization procedure holds until the correlation between multipoles is weak and does not take the measurement errors on the amplitude of the TT spectrum into account. However, we expect that the final results would not be strongly affected by a more accurate normalization. Notice that, as mentioned earlier, $\sigma_{8}$ data is not used to normalize the spectrum. Hence, its value may be evaluated from the matter power spectrum and compared with the observed values.

The weak lensing add-on code (Schimd et al. 2005) manipulates the CMB-code output, notably the angular distance and the 3D power spectrum of the deflecting potential, yielding twopoint correlation functions for the cosmic shear. Once the linear matter power spectrum is known at every redshift, the weak lensing add-on code computes first the non-linear power spectrum using two mappings, by Peacock \& Dodds (1996) and Smith et al. (2003). In particular, we consider wavevectors ranging up to $10 \mathrm{~h} \mathrm{Mpc}^{-1}$ (corresponding to 1 arcmin at $z \sim 1$ for cosmologies close to $\Lambda \mathrm{CDM})$. Then, by Limber projection, it deduces the shear power spectrum allowing for a redshift distribution of sources $n(z)$ parameterized by Eq. (27), calibrated on lensing datasets. Finally, the code computes several two-point statistics in real space, namely the top-hat shear variance $\left\langle\gamma^{2}\right\rangle$, the aperture mass variance $\left\langle M_{\mathrm{ap}}^{2}\right\rangle$, as well as the two-point correlations $\xi_{ \pm}$. Let us stress that, like the Boltzmann code, the lensing add-on code works also with scalar-tensor theories of gravity.

Cosmological parameters are estimated by comparing the predicted signal $s_{i}$ to the data $d_{i}$ as a function of scale $X_{i}$ (which reduces to an angular scale $\theta_{i}$, a redshift $z_{i}$, or a multipole $\ell_{i}$, for lensing, supernovae, and $\mathrm{CMB}$ data, respectively). We vary the

1 After this manuscript was submitted, the WMAP-3yr data were released (Jarosik et al. 2006; Hinshaw et al. 2006; Page et al. 2006; Spergel et al. 2006). According to the normalization procedure we employ, the use of WMAP-3yr instead of WMAP-1yr data would change the normalization factor by less than $1 \%$, a negligible variation provided the actual accuracy of both the weak lensing observed spectra and the linear-to-non-linear mappings we adopt. parameters of the model, disposing them on a regularly spaced grid, and at each grid point evaluate the likelihood function,

$$
\mathcal{L}=\frac{1}{(2 \pi)^{n}|C|^{1 / 2}} \exp \left[-\frac{1}{2}\left(d_{i}-s_{i}\right)^{T} C^{-1}\left(d_{i}-s_{i}\right)\right]
$$

Here $C^{-1}$ is the data covariance matrix, including Poisson shot noise and cosmic variance.

We restrict ourselves to a low-dimensional parameter space and focus on constraining the dark energy density and the parameter $\alpha$ of the quintessence potentials. Ideally, one would include at least nine cosmological parameters: the spatial curvature, the Hubble constant, the parameter $\alpha$ of the potential, the dark energy and matter (both dark matter and baryonic) density parameters, the reionization optical depth, the amplitude, and the spectral index of the initial power spectrum. In addition, one should include the three parameters accounting for the redshift distribution of sources, Eq. (27).

Given the result of the analysis of CMB data (Spergel et al. 2003), we have assumed a spatially flat universe so that $\Omega_{\mathrm{m} 0}=$ $1-\Omega_{\mathrm{Q} 0}$. The amplitude of the initial power spectrum is fixed by the normalization on the CMB. We have also assumed that the reduced Hubble constant, the reionization optical depth, and the baryon energy density are fixed to $h=0.72, \tau_{\text {reion }}=0.17$, and $\Omega_{\mathrm{b} 0} h^{2}=0.024$, respectively.

We preliminarily performed a Fisher matrix analysis on the parameter space $\left(\alpha, \Omega_{\mathrm{Q} 0}, n_{\mathrm{S}}\right)$ in order to estimate the extent of the $1 \sigma$ region and decide the sampling steps of the grid for the computation of the likelihood (notice however that it holds only locally). Considering a synthetic CFHTLS-wide like survey covering $170 \mathrm{deg}^{2}$, with 20 galaxies/arcmin ${ }^{2}$ and an intrinsic ellipticity of 0.4 , we estimate the $68 \%, 95 \%$, and $99 \%$ confidence levels (C.L.) for cosmic shear (top-hat variance), for both Ratra-Peebles and SUGRA models; see Fig. 6. In passing, notice that SUGRA models appear to be more constrained. The fiducial model that is fixed at $\alpha=6, \Omega_{\mathrm{Q} 0}=0.73$, and $n_{\mathrm{s}}=1.0$, while other cosmological parameters are kept fixed, in particular $\left(h, \tau_{\text {reion }}, \Omega_{\mathrm{b} 0} h^{2}\right)=(0.72,0.17,0.024)$. 

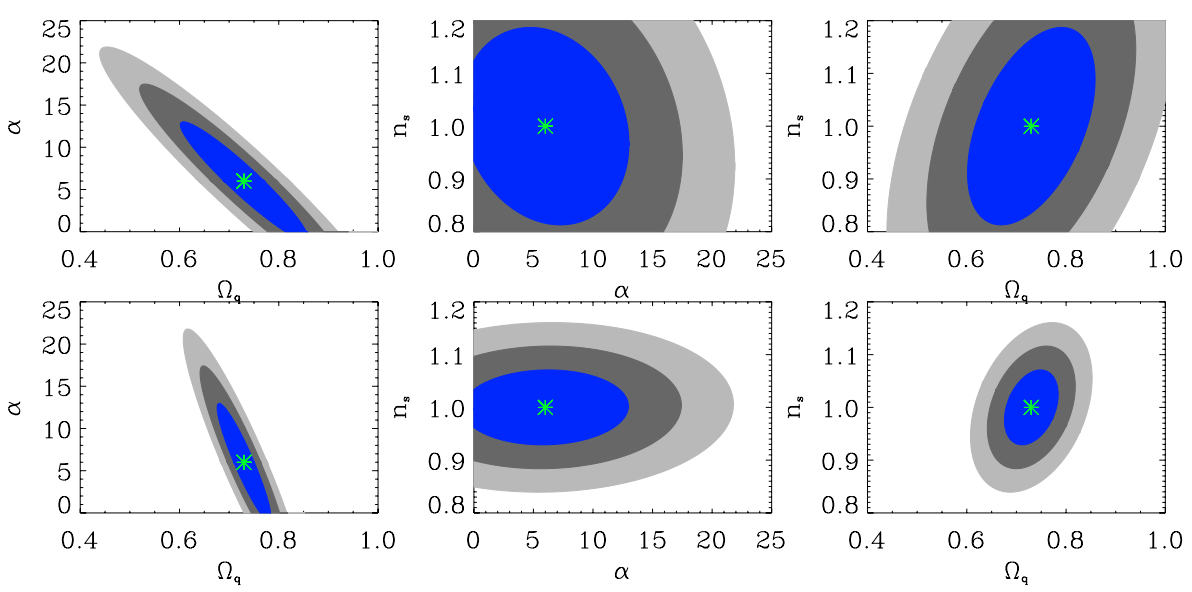

Fig. 6. Fisher analysis of the top-hat shear variance of the cosmic shear for Ratra-Peebles (top line) and SUGRA (bottom line) models. Used to define the grid for the likelihood analysis. It consider a CFHTLS-wide like survey (see text for details), with all but cosmological parameters $\left(\alpha, \Omega_{\mathrm{Q} 0}, n_{\mathrm{s}}\right)$ kept fixed. Contours at 68,95 , and $99 \%$ C.L. See Sect. 3.4 for discussion.

According to this Fisher analysis, in the final likelihood analysis we allow the cosmological parameters to vary in the following ranges

$\Omega_{\mathrm{Q} 0} \in[0.4,0.9], \quad \alpha \in[0,25], \quad n_{\mathrm{s}} \in[0.9,1.1]$.

For the cosmic shear data, we also allow one of the parameters of the source redshift distribution to vary. We choose $z_{\mathrm{s}}$, always marginalized in the final analysis, over its $2 \sigma$ interval; see Eqs. (28) and (29).

Indeed, one can criticize these assumptions but they are sufficient $i$ ) to give us an idea of the parameter space available for dark energy realized by quintessence and ii) to discuss to what extent weak lensing can improve the constraints on dark energy models. From a pragmatic point of view, we were limited by computational capacities so a more complete analysis will follow. Let us stress that such an analysis requires an investigation of the potential degeneracies of the dark energy parameters with the standard cosmological parameters and, in particular, one would need to quantify how allowing for dark energy changes the allowed range of variation of the other cosmological parameters. This is left for further studies.

\subsection{Likelihood analysis: joint cosmic shear data}

Using both real and synthetic cosmic shear data, we perform the likelihood analysis aiming to investigate to what extent the constraints on cosmological parameters and, in particular, the quintessence ones, depend on the linear-to-non-linear mapping and on the selection effects of the two-point statistics.

In this section, we focus on the first issue by combining top-hat variance data of VIRMOS-Descart, CFHTLS-deep, and CFHTLS-wide (22 $\mathrm{deg}^{2}$ sub-sample) surveys; see Table 1 for numerical results of individual parameters constraints. Figure 7 depicts the results quoting the $68 \%, 95 \%$, and $99 \%$ confidence level contours. In particular, we compute the non-linear spectra by both the Peacock \& Dodds (1996) and Smith et al. (2003) procedures, for Ratra-Peebles and SUGRA models as well, hence allowing for two kinds of comparisons.

First, we can compare Ratra-Peebles and SUGRA likelihood contours, disregarding the non-linear mappings. There are two striking differences. One is the strong constraint on $\Omega_{\mathrm{Q} 0}$ found in the SUGRA case. The reason for this is that, since the amplitude of the power spectrum is kept fixed, the $\left(\sigma_{8}, \Omega_{\mathrm{m} 0}\right)$ degeneracy implies a strong constraint on $\Omega_{\mathrm{m} 0}$ and consequently on $\Omega_{\mathrm{Q} 0}$ through the flat universe prior. This effect is stronger in SUGRA models than in Ratra-Peebles ones since the former approach a $\Lambda \mathrm{CDM}$ at low redshift. The other difference is the well-defined degeneracy found in the $\left(\Omega_{\mathrm{Q} 0}, \alpha\right)$ plane for the Ratra-Peebles case. This feature will allow us to put a stronger constraint on Ratra-Peebles' $\alpha$ than in SUGRA's $\alpha$ when combining with other data.

Second and perhaps more interestingly, by comparing the non-linear mappings, two classes of cosmological parameters emerge for both Ratra-Peebles and SUGRA models. Those concerning the quintessence, $\alpha$ and $\Omega_{\mathrm{Q} 0}$, are found to be essentially independent of the non-linear mapping used. This supports the claim of Simpson \& Bridle (2005) that cosmic shear is sensitive to dark energy mostly through the background dynamics. The second class involves the cosmological parameters accounting for the primordial universe, here only $n_{\mathrm{s}}$. In this case the corresponding likelihood contours strongly depend on the chosen mapping, regardless of the quintessence potential we used. Hence it seems impossible to constrain the primordial spectral index, at least jointly with quintessence parameters, until a stable formulation of the non-linear regime of structures formation is available.

\subsection{Likelihood analysis: synthetic data}

Using wide surveys, we can eventually investigate the effect of the non-linear regime of the structures formation. For this purpose, we perform a likelihood analysis using a synthetic realization of the full CFHTLS-wide survey. This consists of synthetic data vectors of top-hat and aperture mass variance and on a synthetic covariance matrix. The former are computed taking as fiducial model a $\triangle \mathrm{CDM}$ with the current CFHTLS-wide redshift distribution. The covariance matrix is computed using the analytical approximation derived in Schneider et al. (2002). It depends on three main features of the survey; the effective area $A$, the effective galaxy number density $n_{\text {gal }}$, and the dispersion of the distribution of ellipticities $\sigma_{\mathrm{e}}$. For these parameters we use the values that are expected at the end of the CFHTLS-wide campaign, respectively: $A=170 \mathrm{deg}^{2}, n_{\text {gal }}=20 \mathrm{gal} / \mathrm{arcmin}^{2}$, and $\sigma_{\mathrm{e}}=0.4$. Notice we assume a larger ellipticity dispersion and a higher density of galaxies than those obtained in Hoekstra et al. (2005). They correspond to a different galaxy-weighting scheme than the one used with current data.

Figures 8 and 9 outline the likelihood analysis of RatraPeebles and SUGRA models, respectively - contours at $68 \%$, $95 \%$, and $99 \%$ confidence level. We show only the results achieved using the mapping by Peacock \& Dodds (1996), those achieved using the mapping by Smith et al. (2003) being in agreement at a $10 \%$ level. 


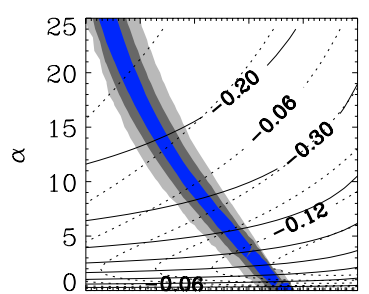

$\begin{array}{llllll}0.4 & 0.5 & 0.6 & 0.7 & 0.8 & 0.9\end{array}$

$\Omega_{\mathrm{q}}$

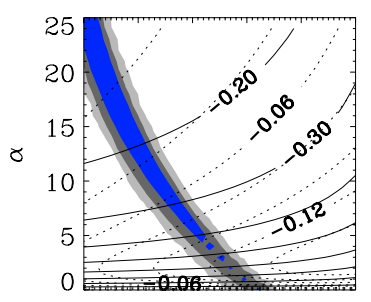

$\begin{array}{lllllll}0.4 & 0.5 & 0.6 & 0.7 & 0.8 & 0.9\end{array}$

$\Omega_{\mathrm{q}}$

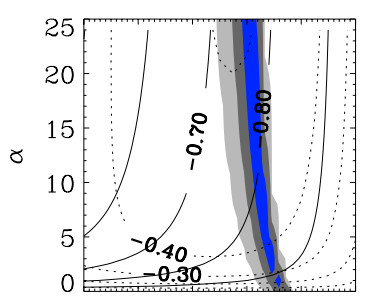

$\begin{array}{llllll}0.4 & 0.5 & 0.6 & 0.7 & 0.8 & 0.9\end{array}$

$\Omega_{\mathrm{q}}$

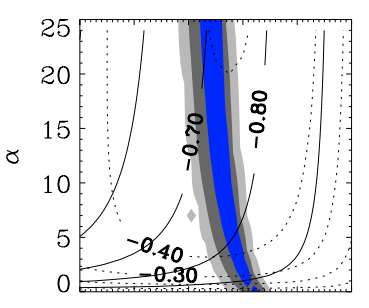

$\begin{array}{llllll}0.4 & 0.5 & 0.6 & 0.7 & 0.8 & 0.9\end{array}$

$\Omega_{\mathrm{q}}$
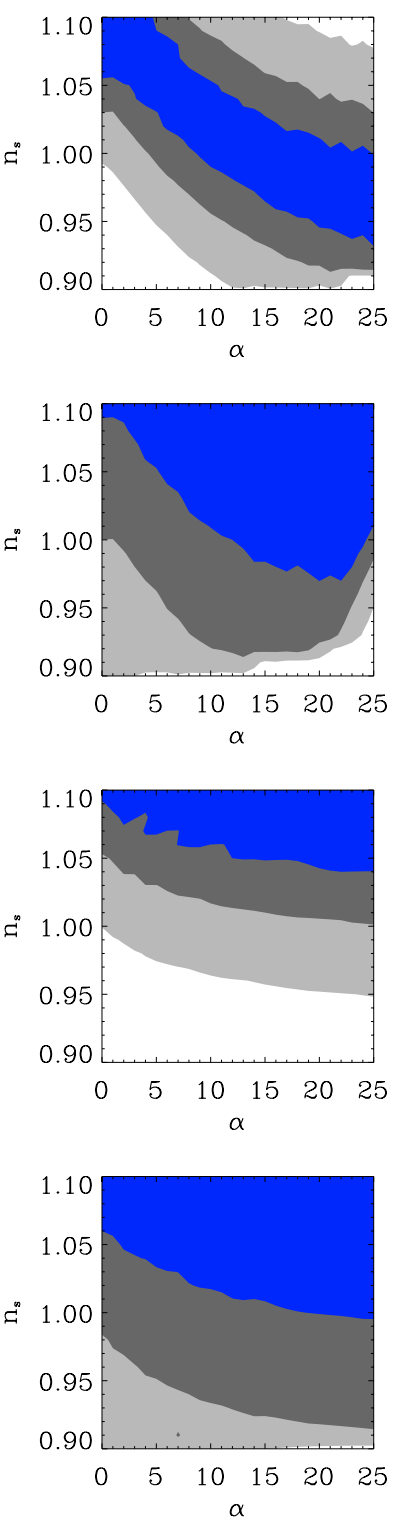

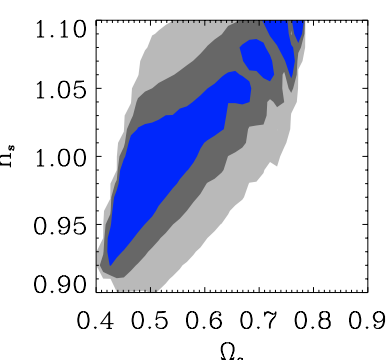

$\Omega_{\mathrm{q}}$
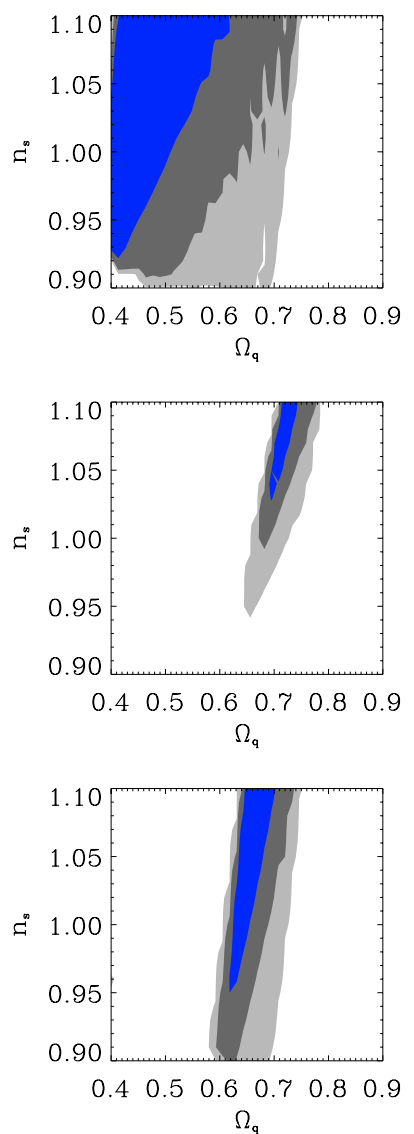

Fig. 7. Joint analysis of VIRMOS-Descart, CFHTLS-deep, and CFHTLS-wide/22 $\mathrm{deg}^{2}$ top-hat variance data (contours at $68 \%, 95 \%$, and $99 \%$ C.L.), marginalizing the likelihood over $z_{\mathrm{s}}$. In the quintessence parameters subspace, the contour lines of $w_{\text {pivot }}$ (solid) and $w_{a}$ (dashed) for $z_{\text {pivot }}=0.5$ are superposed; see Fig. 3. The two upper lines refer to RatraPeebles models, the two lower ones to SUGRA models, $\alpha=0$ corresponding to a $\Lambda \mathrm{CDM}$. For each class of models, the non-linear spectrum has been computed using both the Peacock \& Dodds (1996) procedure, first and third lines, and the halo-model approach by Smith et al. (2003), second and fourth lines. See Sect. 3.5 for discussion.
First, by comparing the results achieved using the top-hat variance data of the $22 \mathrm{deg}^{2}$ sub-sample (first line) with those of the synthetic $170 \mathrm{deg}^{2}$ field (second line), there is an evident gain achievable with the full survey. In particular, it is worth noticing that the pure quintessence parameters sub-space is less dependent on the survey area, while the constraints on the primordial spectral index strongly depend on it. Hence, the distinction of cosmological parameters in two classes introduced in the previous section seems confirmed.

The second and third lines show the likelihood contours for the top-hat and aperture mass variances, respectively. They are consistent for both Ratra-Peebles and SUGRA models, depicting compatible confidence level regions. There are two main differences in the properties of aperture mass and top-hat variances. Measurements of aperture mass variance at different scales are less correlated than top-hat variance ones, since the former is a narrow filtered version of the shear power spectrum; and their effective range extends to only about $1 / 5$ of the top-hat variance one. They thus follow more accurately the shape of the power spectrum at their measured range. For a featureless power spectrum on most scales but these, the independent measurements make this statistic the most convenient to use. In this case, contours from aperture mass variance are expected to be smaller than top-hat variance ones. But in general, the loss of information regarding the behaviour of models on other scales diminishes the capability of distinguishing between models. Consequently, the aperture mass variance contours are, in general, expected to be larger than top-hat variance ones. The difference is larger when analysing most important parameters of the cosmic shear. Since we are studying those parameters that cosmic shear is moderately sensitive to, the difference is not noticeable in our results in Figs. 8 and 9, but it is striking when using VIRMOS-Descart data to constrain the $\left(\sigma_{8}, \Omega_{\mathrm{m}}\right)$ plane (van Waerbeke et al. 2001). Furthermore, for our data with measurements up to less than $1 \mathrm{deg}$, the largest power spectrum scale probed by the aperture mass variance is the one probed by the top-hat variance at around 10 arcmin and its full range effectively lies on non-linear scales, rendering its infered parameters' constraints less reliable, due to non-linear modeling uncertainties. For these two reasons, we choose to use only top-hat variance, when producing the final results.

By using the full wide survey, we can try to disentangle the effects of the non-linear regime of structures formation by cutting off the small angular scales from the final analysis. 


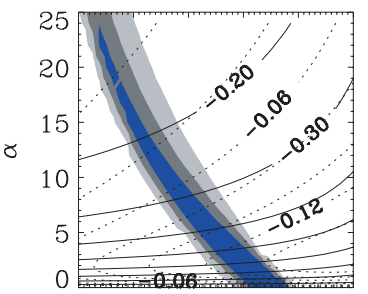

$\begin{array}{llllll}0.4 & 0.5 & 0.6 & 0.7 & 0.8 & 0.9\end{array}$

$\Omega_{\mathrm{q}}$

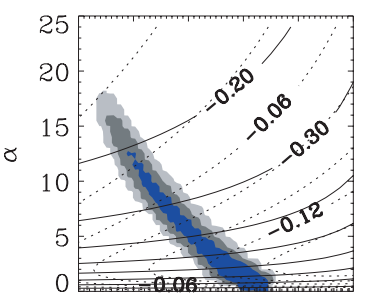

$\begin{array}{llllll}0.4 & 0.5 & 0.6 & 0.7 & 0.8 & 0.9\end{array}$ $\Omega_{\mathrm{o}}$

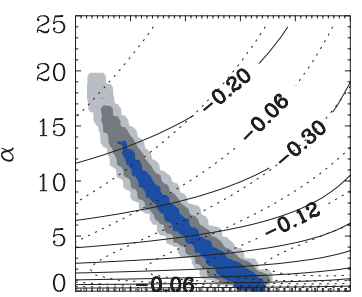

$\begin{array}{llllll}0.4 & 0.5 & 0.6 & 0.7 & 0.8 & 0.9\end{array}$ $\Omega_{\mathrm{q}}$

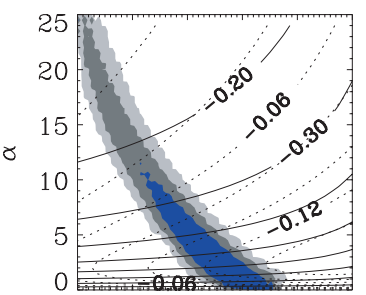

$\begin{array}{llllll}0.4 & 0.5 & 0.6 & 0.7 & 0.8 & 0.9\end{array}$ $\Omega_{\mathrm{q}}$
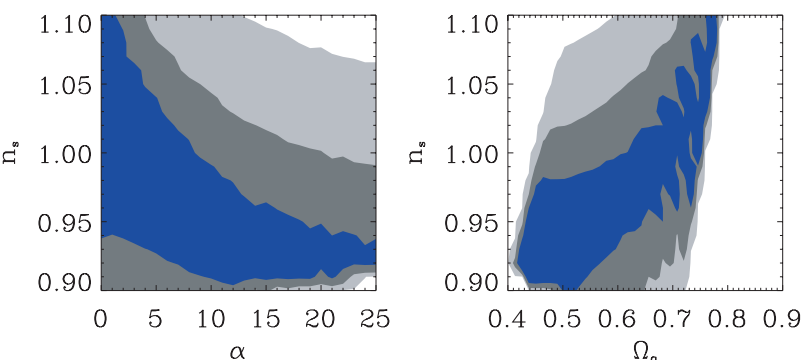

$\begin{array}{llllll}0.4 & 0.5 & 0.6 & 0.7 & 0.8 & 0.9\end{array}$

$\Omega$
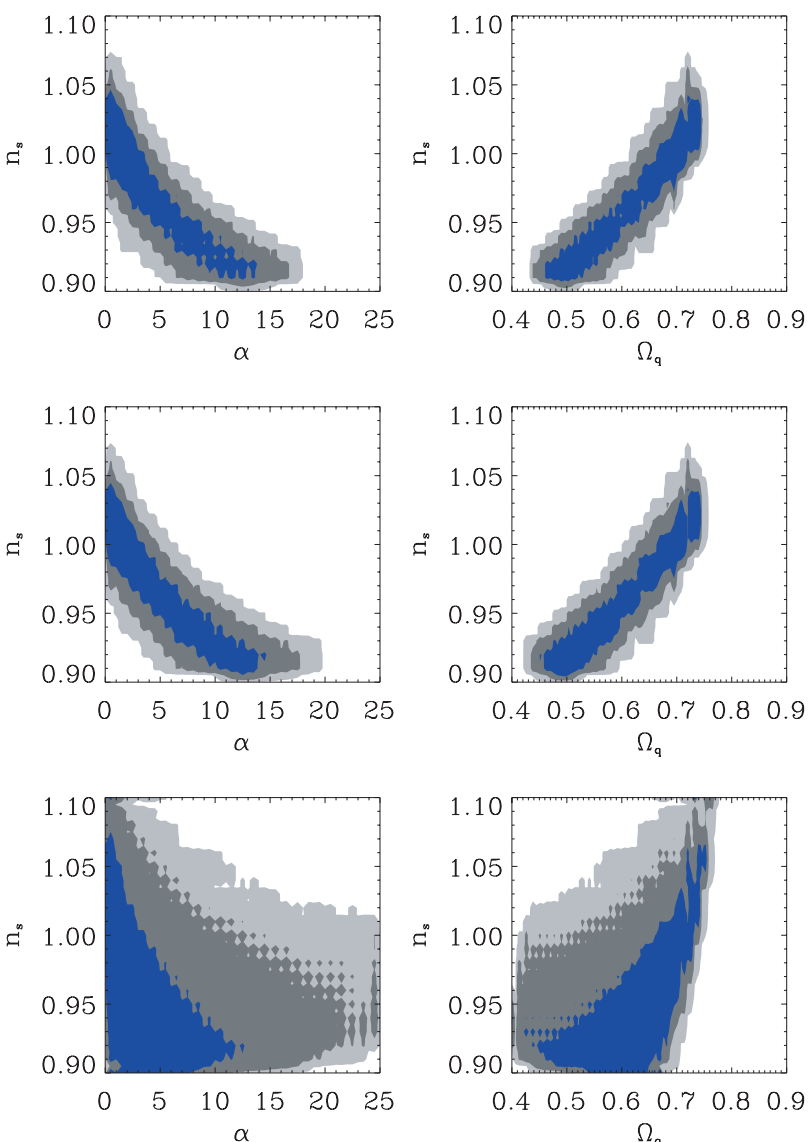

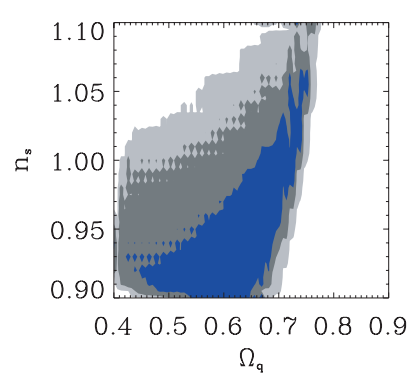

Fig. 8. CFHTLS-wide constraints on RatraPeebles models (contours 68, 95, and 99\% C.L.) marginalizing over $z_{\mathrm{s}}$. As before, in the quintessence parameters sub-space the contour lines of $\left(w_{\text {pivot }}, w_{a}\right)$ for $z_{\text {pivot }}=0.5$ are superposed. The first line depicts the analysis of the actual dataset based on $22 \mathrm{deg}^{2}$ (W1+W3; see Sect. 3.6), while the three other lines are based on synthetic data for a field of $170 \mathrm{deg}^{2}$. The second and third lines show the top-hat shear variance and aperture mass variance, respectively. The fourth line describes the analysis of the same simulated data for the top-hat shear variance, but using only angular scales larger than 20 arcmin to cut out the non-linear part of the matter power spectrum. Here the Peacock \& Dodds (1996) mapping for the nonlinear power spectrum is used. See Sect. 3.6 for discussion.
In such a way, we can better investigate the distinction of cosmological parameters in the two classes discussed above, probing whether dark energy primarily hangs on the background dynamics. The plots on the bottom lines of Figs. 8 and 9 depict the analysis of the top-hat variance when taking into account only angular scales larger than 20 arcmin, corresponding to wavevectors $k \lesssim 1 h \mathrm{Mpc}^{-1}$ at $z \lesssim\langle z\rangle / 2 \sim 0.5$, where the effects of the non-linear regime are therefore sub-dominant. The spread in the likelihood contours is more relevant for plots involving the primordial spectral index $n_{\mathrm{s}}$, while the quintessence parameters $\left(\alpha, \Omega_{\mathrm{Q} 0}\right)$ are not affected as much. Hence, one can study dark energy by cosmic shear using this technique even if not certain of how to deal with the non-linear regime. Obviously this conclusion has to be confirmed by a more complete study involving a larger parameter space, to account for other degeneracies.

The first lines of Figs. 8 and 9 show the current results of CFHTLS-wide, using W1 and W3 data from an effective sky coverage of $22 \mathrm{deg}^{2}$. The corresponding marginalized constraints on $\alpha$ and $\Omega_{\mathrm{Q} 0}$ are a factor of 2 larger than the ones found with the synthetic data, depicted on the second line of the same figures. This is consistent with the reduction in the data error bars, which are proportional to $\sigma_{\mathrm{e}}^{2} n_{\mathrm{gal}}^{-1} A^{-1 / 2}$, if cosmic variance is not taken into account. We must caution that the gain in the parameter space cannot be estimated with precision by this simple argument, namely by assuming

gain $\sim \frac{r_{\sigma_{\mathrm{e}}}^{2}}{r_{n_{\mathrm{gal}}} \sqrt{r_{A}}}$,

where the several factors $r$ are the ratios between the features of the two surveys. In fact, the gain in the data error bars does not translate linearly into a gain in the parameters space confidence levels. That happens only in the Fisher matrix approximation and even there, only in the case of statistical uncorrelated parameters. Furthermore, this reasoning does not take into account the extra constraining power coming from measurements on larger scales, as discussed earlier on, or simply coming from the fact of disposing of more degrees of freedom for the likelihood calculations.

It is also worthwhile to notice that, in all cases (see Figs. 7 to 9), a $\Lambda$ CDM model with a Harrison-Zel'dovich spectrum $\left[\left(\alpha, n_{\mathrm{s}}\right)=(0,1)\right]$ is compatible with the data for $\Omega_{\mathrm{Q} 0} \sim 0.7$ at a $99 \%$ confidence level. 


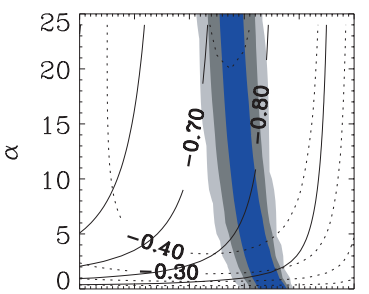

$\begin{array}{llllll}0.4 & 0.5 & 0.6 & 0.7 & 0.8 & 0.9\end{array}$ $\Omega_{\mathrm{q}}$

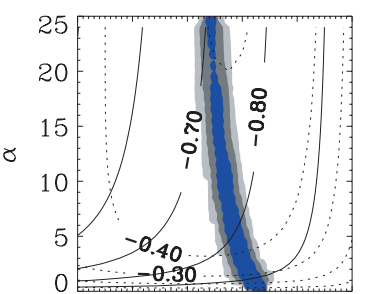

$\begin{array}{llllll}0.4 & 0.5 & 0.6 & 0.7 & 0.8 & 0.9\end{array}$ $\Omega_{\mathrm{q}}$

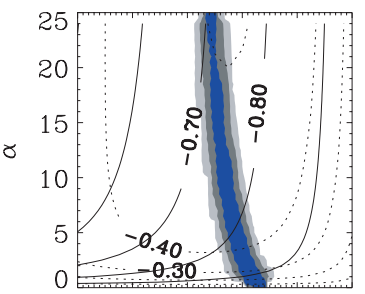

$\begin{array}{llllll}0.4 & 0.5 & 0.6 & 0.7 & 0.8 & 0.9\end{array}$ $\Omega_{\mathrm{q}}$

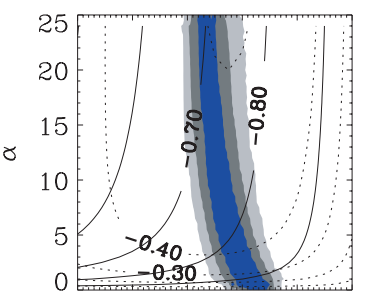

$\begin{array}{llllll}0.4 & 0.5 & 0.6 & 0.7 & 0.8 & 0.9\end{array}$ $\Omega_{\mathrm{q}}$
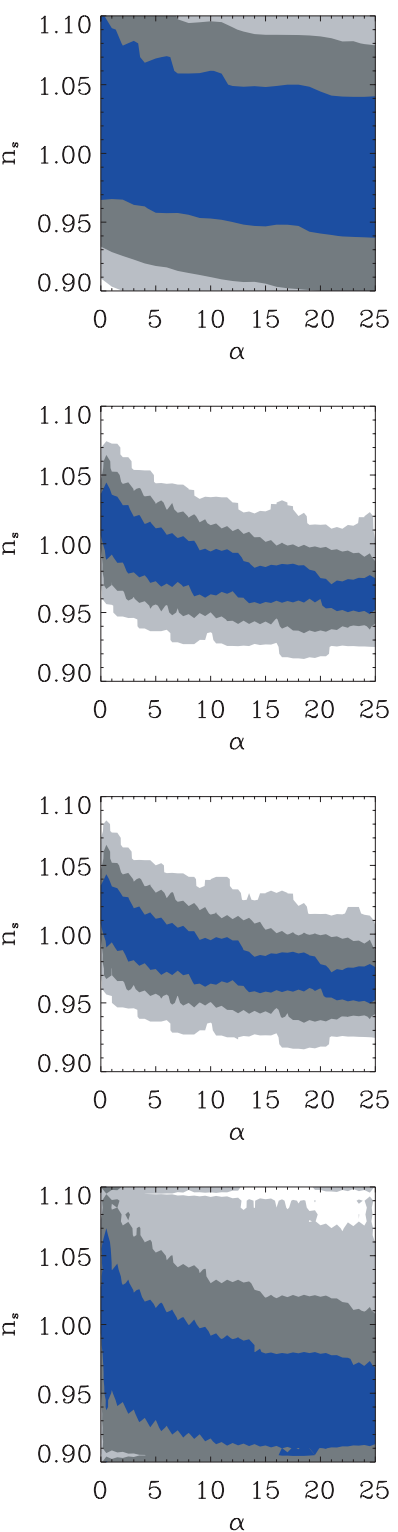

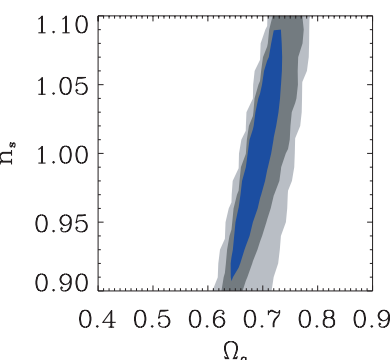

$\Omega$

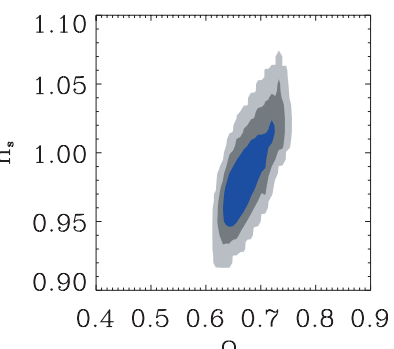

$\Omega_{\mathrm{q}}$
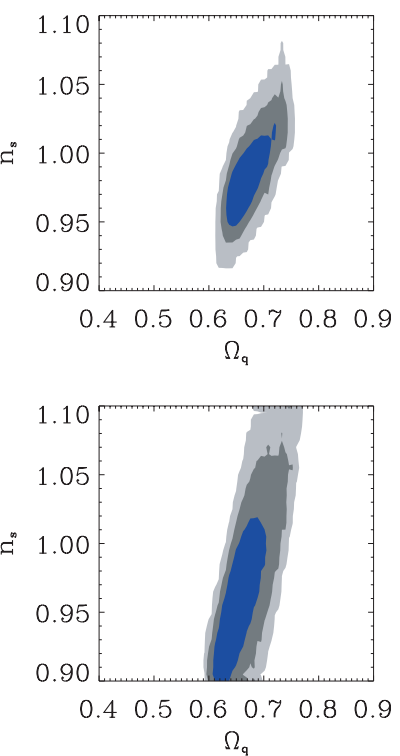

Fig. 9. CFHTLS-wide constraints on SUGRA models. See the analogous Fig. 8 for the caption.

\section{Combining with other observables}

The computational tools we developed allow us to compute distance modulus, CMB anisotropies, and weak lensing - cosmic shear effects in the same framework. In principle there is therefore no problem combining Sn Ia, CMB and weak lensing data. As already noticed, we are restricted here to a small parameter space, so only a joint analysis of Sn Ia and weak lensing data is possible (Sect. 4.1). Indeed, a joint analysis with CMB data requires a wider parameter space, possibly using the so-called "normal parameters" (see e.g. Sandvik et al. 2004), an option left for a future study. Here we restrict the use of CMB data to the normalization of the power spectrum and to excluding a region of the parameter space (Sect. 4.2).

\subsection{Sn la}

We combine the cosmic shear data by VIRMOS-Descart and CFHTLS-deep and -wide (22 $\mathrm{deg}^{2}$ sub-sample) surveys with the type Ia supernovae "gold" set by Tonry et al. (2003). In particular, we evaluate confidence intervals considering cosmic shear and Sn Ia both separately and jointly. Indeed, since the distance modulus depends only on the background dynamics, we can restrict the study to the quintessence cosmological parameters $\alpha$ and $\Omega_{\mathrm{Q} 0}$.

Figure 10 depicts both the independent and combined analyses for Ratra-Peebles and SUGRA models, using the top-hat variance data for the cosmic shear and computing the non-linear spectrum by the Peacock \& Dodds (1996) mapping. The corresponding results are summarised in Table 1; these marginalized results for each parameter do not assume any prior knowledge of the other, apart from the flat priors implied by the range of the grid. Let us emphasise that the constraints obtained on $\left(w_{0}, w_{a}\right)$, inferred from those on the parameters of the potential, differ when we change the form of the potential. This confirms that constraints on the equation of state derived from a general parameterization have to be interpreted with care.

Concerning Ratra-Peebles models, the weak lensing and Sn Ia contours are closer to mutual orthogonality, so narrow joint constraints are expected. Data strongly favour a quintessence component close to a cosmological constant, the best fit lying always at $\alpha=0$, so that in Table 1 we prefer to present the constraints as an upper limit. As is clear from Fig. 10, Sn Ia are much more constraining for this parameter than is weak lensing. 

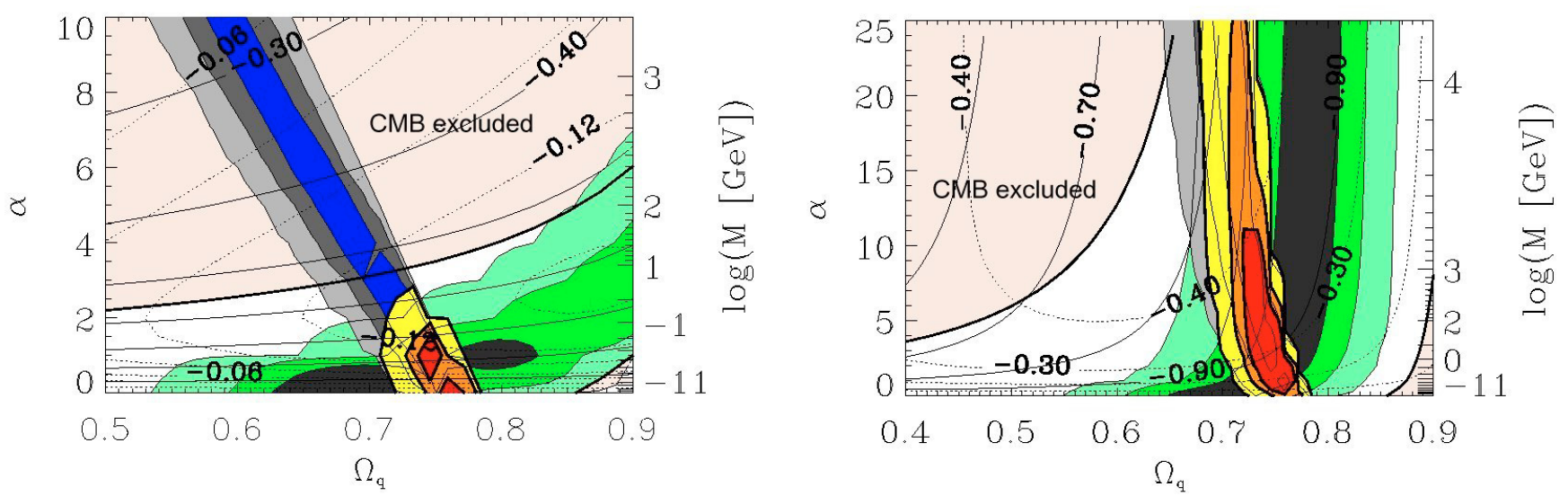

Fig. 10. Joint analysis of the quintessence parameters $\left(\Omega_{\mathrm{Q} 0}, \alpha\right.$, ) from CMB, Sn Ia, and cosmic shear (top-hat variance), for Ratra-Peebles models (left panel) and SUGRA models (right panel); the corresponding constraints are given in Table 1 . The likelihood analysis uses the "gold" set for Sn Ia (green contours) and VIRMOS-Descart + CFTHLS-deep + CFTHLS-wide(22 $\mathrm{deg}^{2}$ ) top-hat variance data for the cosmic shear (blue contours), eventually combined (red contours). Contours correspond to 68, 95, and 99\% C.L. The shaded region is excluded by the location of the first acoustic peak of the temperature CMB power spectrum, according to WMAP-1yr data; see Sect. 4.2 for details. On the right axes, an indicative mass scale $M$ of the quintessence potential is quoted; see Sect. 2 for details. Contours of $w_{\text {pivot }}$ (solid) and $w_{a}$ (dotted) for $z_{\text {pivot }}=0$ are superposed.

Table 1. Results of separate and joint analysis of cosmic-shear and Sn Ia data on quintessence parameters $\left(\alpha, \Omega_{\mathrm{Q} 0}\right)$ at $68 \%$ (95\%) confidence level. For Ratra-Peebles models, we quote only upper limits for $\alpha$, the best fit is always at $\alpha=0$. Only for indicative purposes, we quote $68 \%$ confidence level limits on $\left(w_{\text {pivot }}, w_{a}\right)$ parameters computed at $z_{\text {pivot }}=0$, setting $n . c$. when not constrained. Recall that in this class of models $w_{0} \geq-1$. See Sect. 4.1 for discussion.

\begin{tabular}{lcccc|cccc}
\hline \hline & \multicolumn{4}{c}{ Ratra-Peebles } & \multicolumn{5}{c}{ SUGRA } \\
& $\alpha$ & $\Omega_{\mathrm{Q} 0}$ & $w_{0}$ & $w_{a}$ & $\alpha$ & $\Omega_{\mathrm{Q} 0}$ & $w_{0}$ & $w_{a}$ \\
\hline$\left\langle\gamma^{2}\right\rangle$ & $<14(25)$ & $0.63_{-0.11(+0.15)}^{+0.14(+0.20)}$ & n.c. & n.c. & $13_{-5(-13)}^{+12(+12)}$ & $0.72_{-0.02(-0.03)}^{+0.01(+0.03)}$ & $\lesssim-0.79$ & $\gtrsim-0.45$ \\
Sn Ia & $<1(3)$ & $0.74_{-0.10(+0.16)}^{+0.09(+0.11)}$ & $\lesssim-0.75$ & $\gtrsim-0.13$ & $12_{-12(-12)}^{+5(11)}$ & $0.77_{-0.04(-0.07)}^{+0.04(+0.07)}$ & $\lesssim-0.84$ & $\gtrsim-0.43$ \\
$\left\langle\gamma^{2}\right\rangle+$ Sn Ia & $<1(1)$ & $0.75_{-0.04(-0.04)}^{+0.02(+03)}$ & $\lesssim-0.7$ & $\gtrsim-0.13$ & $2_{-1(-2)}^{+7(+18)}$ & $0.74_{-0.04(-0.05)}^{+0.03(+0.03)}$ & $\lesssim-0.84$ & $\gtrsim-0.38$ \\
\hline
\end{tabular}

However, even though Sn Ia alone reject $\alpha \geq 1$ at the $68 \%$ level, as was well known (Podariu \& Ratra 2000), the information from weak lensing further narrows the interval.

As for SUGRA models, for a wide interval of $\alpha$ (approximately $\alpha>5$ ) both the luminosity distance and the shear twopoint correlations are (almost) independent of this parameter, as is clear from Fig. 10. In fact, this conclusion has been already reached by studying the CMB temperature anisotropies (Brax et al. 2000), arguing that the equation of state does not strongly depend on the slope of the potential leaving both distances and the linear growth factor almost unchanged. In terms of statistical significance, this means that the likelihood with respect to $\mathrm{Sn}$ Ia or weak lensing data alone is almost flat and so the best-fit value has little meaning (see also Caresia et al. 2004). In Table 1 we list the results in the form of a parameter value, which we take to be the likelihood-weighted average of $\alpha$, plus or minus the necessary deltas to form the confidence intervals obtained. However, for a substantial interval of $\alpha$, weak lensing and Sn Ia lead to different, almost non-intersecting, ranges of $\Omega_{\mathrm{Q} 0}$ : Sn Ia favouring a higher value of $\Omega_{\mathrm{Q} 0}$ and weak lensing a lower one. Thus, even though both observables have a limited sensitivity to constrain the parameter $\alpha$, this fact allows us to obtain a reasonable constraint from the joint likelihood, as that interval of $\alpha$ is rejected by the data. The joint confidence intervals are substantially reduced, with a distinctive maximum of likelihood at $\alpha=2$. Notice, however, that this is an unstable situation. In fact, if cosmic shear or supernovae contours slightly change their orientation and size, due for example to a larger uncertainty on the redshift of sources, the joint contour will easily degrade. In contrast, the extreme situation, with both contours vertical and parallel for all $\alpha$, would imply the abandon of the subjacent quintessence model. Every systematic effect on weak lensing or supernovae data, relying for instance on the data analysis procedure, also strongly affects the final result; therefore, special care is necessary when combining several datasets.

Provided that it is not evident which pivot redshift should be used when parameterizing these quintessence models by an equation of state of the form of Eq. (13), we superpose to the $\left(\Omega_{\mathrm{Q} 0}, \alpha\right)$ plane, in Fig. 10, the contours for $w_{\text {pivot }}$ and $w_{a}$ corresponding to $z_{\text {pivot }}=0$. The final results in terms of the equation of state parameters are only indicative. Interestingly, notice from Fig. 3 that for Ratra-Peebles models the estimation of $w_{\text {pivot }}$ and $w_{a}$ should not change if using $z_{\text {pivot }}=0.5$ instead of $z_{\text {pivot }}=0$. In fact, for this class of models, noticeably different values on these parameters only appear when considering a high pivot redshift. On the contrary, for SUGRA models the choice of the pivot redshift would already be relevant at low redshift. See Table 1 for specific constraints on $w_{0}$ and $w_{a}$.

\section{2. $C M B$}

CMB temperature anisotropies have been used extensively (see e.g. Baccigalupi et al. 2002; Jassal et al. 2004; Corasaniti et al. 2004) but several degeneracies amongst the cosmological parameters prevent accurately constraining the cosmological parameters using only $\mathrm{CMB}$ data. Hence several studies using weak lensing already attempt to combine $\mathrm{CMB}$ and cosmic shear 

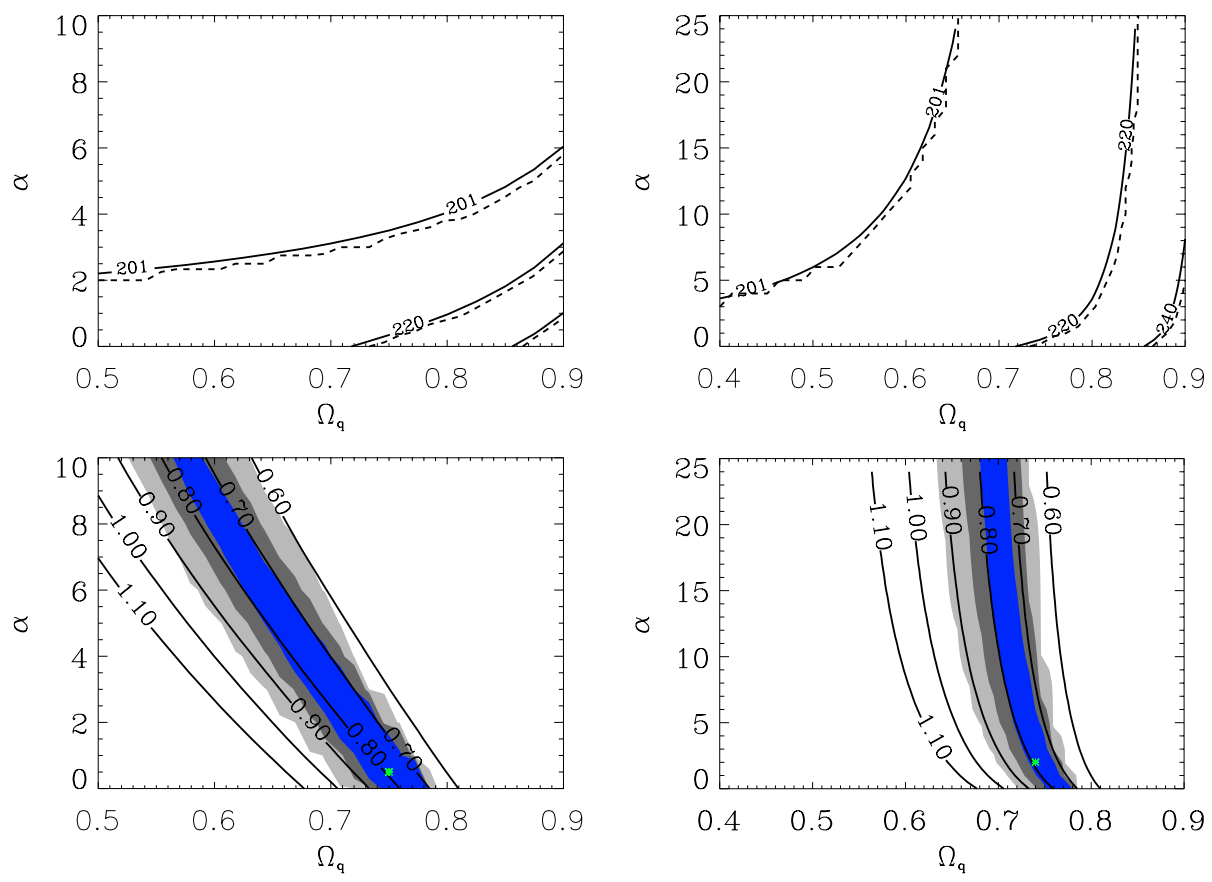

Fig. 11. Top line: Location of the first acoustic peak of the temperature CMB power spectrum for Ratra-Peebles (left panel) and SUGRA (right panel) models, estimated using an analytical approximation (solid line) or by the full computation of $C_{\ell}$ s (dashed line). The central set of lines corresponds to the location of the first peak according to the best-fit of WMAP$1 \mathrm{yr}$ data, $\ell=220.1 \pm 0.8$ (Page et al. 2003), while the left and right ones correspond to the multipoles $\ell=201$ and $\ell=240$, defining the smallest and the largest multipoles contributing to the two points of binned data defining the first peak (Hinshaw et al. 2003. Results compatible with WMAP-3yr data; see Hinshaw et al. 2006). Bottom line: contour levels of $\sigma_{8}$ for Ratra-Peebles (left panel) and SUGRA (right panel) models with $n_{\mathrm{s}}=1$, superposed to the cosmic shear data contours (VIRMOS-Descart + CFHTLS-deep + CFHTLS-wide/22 $\mathrm{deg}^{2}$ ). For indicative purposes, the star point marks the bestfit from the joint cosmic shear and SN Ia analysis, roughly sitting at $\sigma_{8}=0.8$. See Sect. 4.2 for details. to constrain the cosmological parameters (see e.g. Contaldi et al. 2003; Ishak et al. 2004; Tereno et al. 2004).

Dark energy affects the CMB anisotropies' angular power spectrum at least in two ways (Brax et al. 2000). First, the angular diameter distance is modified so that the peak structure is shifted. In particular, the location of the first acoustic peak provides an estimate of the angular diameter distance to the last scattering surface, depending on the geometry of the universe. However, pre-recombination effects can also shift the peaks from their true geometrical locations (Doran et al. 2001b; Kamionkowski \& Buchalter 2000). Second, the time evolution of the dark energy strongly affects the integrated Sachs-Wolfe effect. This effect is more relevant at low multipoles, modifying the amplitude of the spectrum, but it also leads to an additional shift in the Doppler peaks.

Besides using CMB data to normalize the power spectra (see Sect. 3.4), we use them in still another way. By noticing that even without a statistical analysis of CMB data, we can strongly constrain cosmological parameters, and notably quintessence ones, simply by using the location of the first Doppler peak. Moreover, even without solving the perturbations equations and computing the TT spectrum, one can compute the acoustic scale just by solving the equation for the background evolution. The location of acoustic peaks is then estimated by allowing for the shifts induced by the dark energy with a fitting formulae (Doran \& Lilley 2002). In conclusion, the location of the first acoustic peak will be a function of the quintessence parameters $\left(\Omega_{\mathrm{Q} 0}, \alpha\right)$, with a negligible dependence on the primordial spectral index $n_{\mathrm{s}}$ due to the shift correction. Using this analytic approximation (see Fig. 10), we individuate the region of the $\left(\Omega_{\mathrm{Q} 0}, \alpha\right)$ plane compatible with the location of the first acoustic peak of WMAP-1yr data (Hinshaw et al. 2003; Page et al. 2003) including the bins' contributions to the two points defining the peak, namely $201<$ $\ell<240^{2}$. Consistent with the normalization procedure we used, this result is in excellent agreement with that deduced by the

${ }^{2}$ The thus-defined exclusion region in the $\left(\Omega_{\mathrm{Q} 0}, \alpha\right)$ plane does not change if using WMAP-3yr data (Hinshaw et al. 2006) instead of complete computation of the TT spectrum, as shown in Fig. 11 (top panels). The region of the parameter space $\left(\Omega_{\mathrm{Q} 0}, \alpha\right)$ compatible with the position of the first acoustic peak is degenerate with the Sn Ia constraints, beacause since the pre-recombination effects of quintessence on the definition of the acoustic horizon at last scattering are negligible, the acoustic scale eventually depends only on the Hubble parameter like the luminosity distance; hence, both are ultimately affected by quintessence approximatively in the same way.

As mentioned before, we may compute the $\sigma_{8}$ value a posteriori for each CMB normalized model. The obtained $\sigma_{8}$ isocontours in the $\left(\Omega_{\mathrm{Q} 0}, \alpha\right)$ plane are shown in Fig. 11 (bottom panels), setting $n_{\mathrm{s}}=1$. The $\sigma_{8}$ range that corresponds to the confidence levels found in this work agrees with current cosmic shear $\sigma_{8}$ constraints; see Hoekstra et al. (2005) for a recent result and van Waerbeke \& Mellier (2003) for a compilation of results. This shows that the normalizations on the CMB and at $z=0$ are compatible. It is interesting to note that the directions of the $\Omega_{\mathrm{Q} 0}-\alpha$ degeneracy for lensing data are lines of constant $\sigma_{8}$. As the curvature of the universe is kept fixed, a strong constraint on $\sigma_{8}$ implies a strong constraint on $\Omega_{\mathrm{Q} 0}$, through the well-known $\sigma_{8}-\Omega_{\mathrm{m} 0}$ degeneracy, but only if $\Omega_{\mathrm{Q} 0}$ and $\alpha$ are not correlated much. This is what we observe in the SUGRA case. In general, a contour in the $\left(\sigma_{8}, \Omega_{\mathrm{m}}\right)$ plane will move as a function of $\alpha$ and a strong constraint on $\sigma_{8}$ does not necessarily imply a strong constraint on $\Omega_{\mathrm{Q} 0}$. This is what is obtained for the Ratra-Peebles case.

\section{Weak lensing: prospects for future data}

Beyond CFHTLS, several next-generation cosmic shear surveys have been proposed to resolve $w$ and the dark energy properties in very fine detail. It is interesting to explore the capabilities of such surveys to constrain the models discussed in this paper.

WMAP-1yr data; in both the datasets, the smallest and largest multipoles contributing to the two points defining the first acoustic peak are the same. 

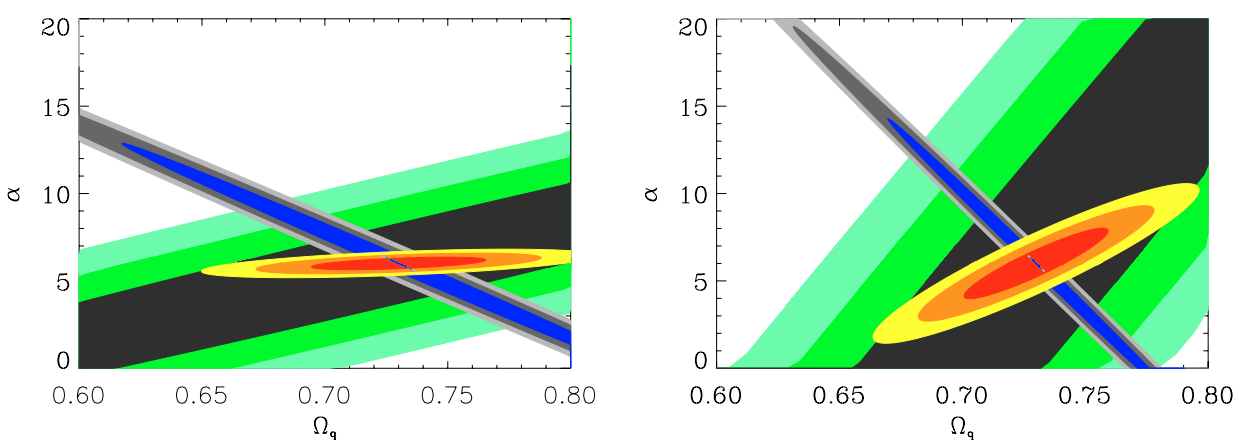

Fig. 12. Fisher analysis for Ratra-Peebles (left) and SUGRA (right) models, for cosmic shear top-hat variance (blue ellipses), Sn Ia "gold" set (green), and temperature CMB angular power spectrum by WMAP-1yr data (red) - contours at 68\%, 95\%, and 99\% C.L., all but $\left(\Omega_{\mathrm{Q}}, \alpha\right)$ parameters kept fixed. For cosmic shear, outer ellipses refer to a CFHTLS-wide like survey $\left(A=170 \mathrm{deg}^{2}, n_{\mathrm{gal}}=20 \mathrm{gal} / \mathrm{arcmin}^{2}, \sigma_{\mathrm{e}}=0.4\right)$, while inner ellipses to a type II space-based survey $\left(A=20000 \mathrm{deg}^{2}, n_{\text {gal }}=35 \mathrm{gal} / \mathrm{arcmin}^{2}, \sigma_{\mathrm{e}}=0.3\right)$. See Sect. 5 for discussion.

A comprehensive study of all projects and observing strategies is, however, beyond the scope of this work, so we deliberately focus on rather simple concepts where both sky coverage and depth are increased, assuming systematics related to shape measurement can be discarded. In particular, we consider two possible layouts achievable by space-based missions, which we indicate by

- type I, a deep survey that would cover about $1000 \mathrm{deg}^{2}$, one magnitude deeper than the CFHTLS-wide one, providing an effective galaxy number density around $50 \mathrm{gal} / \mathrm{arcmin}^{2}$;

- type II, a wider but shallow survey, covering $20000 \mathrm{deg}^{2}$ and yielding $35 \mathrm{gal} / \mathrm{arcmin}^{2}$.

Furthermore, we assume an intrinsic ellipticity distribution of galaxies similar to the one observed with current cosmic shear surveys with HST.

A Fisher analysis of Ratra-Peebles and SUGRA models, restricted to the parameters $\left(\Omega_{\mathrm{Q} 0}, \alpha\right)$, keeping the others fixed, was performed around a fiducial model defined by $\left(\alpha, \Omega_{\mathrm{Q} 0}, n_{\mathrm{s}}, h, \tau_{\text {reion }}, \Omega_{\mathrm{b} 0} h^{2}\right)=(6,0.73,1,0.72,0.17,0.024)$. The results are presented in Fig. 12. Weak-lensing surveys (blue ellipses), together with Sn Ia "gold" set (green ellipses) and CMB WMAP-1yr data (red ellipses; noise matrix computed using the public code by Verde et al. 2003), show that both quintessence parameters can in principle be determined with $10 \%$ accuracy. The degeneracy with respect to $\mathrm{Sn}$ Ia and CMB would be almost totally broken. Is is worth noticing that, since all cosmological parameters but the quintessence ones are kept fixed, we have to be somewhat suspicious of the astonishing gain with respect to a CFHTLS-wide-like survey (larger ellipses) achievable by a space mission of type II (smaller ellipses).

On scales larger than 10 degrees, the flat-sky approximation used in Sect. 3.1 does not hold any longer. Indeed, an angular distance of 15 degrees on a sphere has a $1 \%$ deviation from the same distance on a plane, and spherical harmonics must be considered for larger scales (Stebbins 1996). These effects are not taken into account in the results of Fig. 12, where the gain observed between the two weak-lensing ellipses essentially corresponds to Eq. (30), together with contributions from the fact that measurements from two surveys observing at different scale ranges have different cosmic variances and different degrees of freedom when fitting models to the data. In particular, a strategy of dividing the covered surveys areas in patches of $100 \mathrm{deg}^{2}$ is assumed. Hence, given the high ratio between both sky coverages, this is the dominant factor in the gain.

For indicative purposes, and allowing for the effect of systematic discussed in Sect. 4.1, we restrict the Fisher analysis of weak lensing (top-hat variance) to Ratra-Peebles models, considering a CFHTLS-wide-like survey and both the space-based surveys of types I and II; see Fig. 13. As in Sect. 4.1, we evaluate the parameter space $\left(\alpha, \Omega_{\mathrm{Q} 0}, n_{\mathrm{s}}\right)$. In the upper line, analogous to the likelihood analysis of Sect. 3 that also assumes all the other cosmological parameters fixed, we compare the CFHTLSwide-like survey (wider ellipses) with the space-based surveys of type I (smaller ellipses). The middle line is analogue, but it compares space-based surveys of type I (wider ellipses) with the space-based surveys of type II (smaller ellipses); notice that the apparent rotation of the ellipses is simply due to a rescaling of the axes. As for quintessence parameters, a space-based survey of type II gives $99 \%$ C.L. contours, approximatively 3 times smaller than those achievable by a type I survey. Indeed more cosmological parameters have to be taken into account. As an example, allowing the reionization optical depth $\tau_{\text {reion }}$ to vary, the likelihood contours get strongly modified; see the third line of Fig. 13, where we compare a CFHTLS-wide like and type II space-based surveys, marginalizing over $\tau_{\text {reion }}$. They get larger and the degeneracies directions are changed. The strong impact of the reionization optical depth in cosmic-shear results comes from the degeneracy with between $\tau_{\text {reion }}$ and the normalization of the spectrum.

The great predictive power of space-based surveys will allow us to simultaneously constrain a large number of parameters with good precision, even if not so high as the one showed in the analysis of a small number of parameters. However, it is important to notice that we have not taken into account the possible use of tomography based on a decomposition of lensing data into several lensed/source planes, nor any use of higher-order statistics than the top-hat shear variance. Even if several issues have been neglected in the present study, our conclusions are very likely not over-optimistic.

\section{Conclusions}

In this article that uses VIRMOS-Descart and CFHTLS data for the first time to study dynamical dark energy models, we have investigated the constraints set by the cosmic shear on two families of quintessence models, illustrating the complementarity of these observations with other data sets, notably supernovae and CMB.

In such a situation where a physical model is fully specified, we can treat both the background and perturbation evolution without any ambiguity, without specifying an analytical form for the transfer function. Such an approach is thus suitable for 

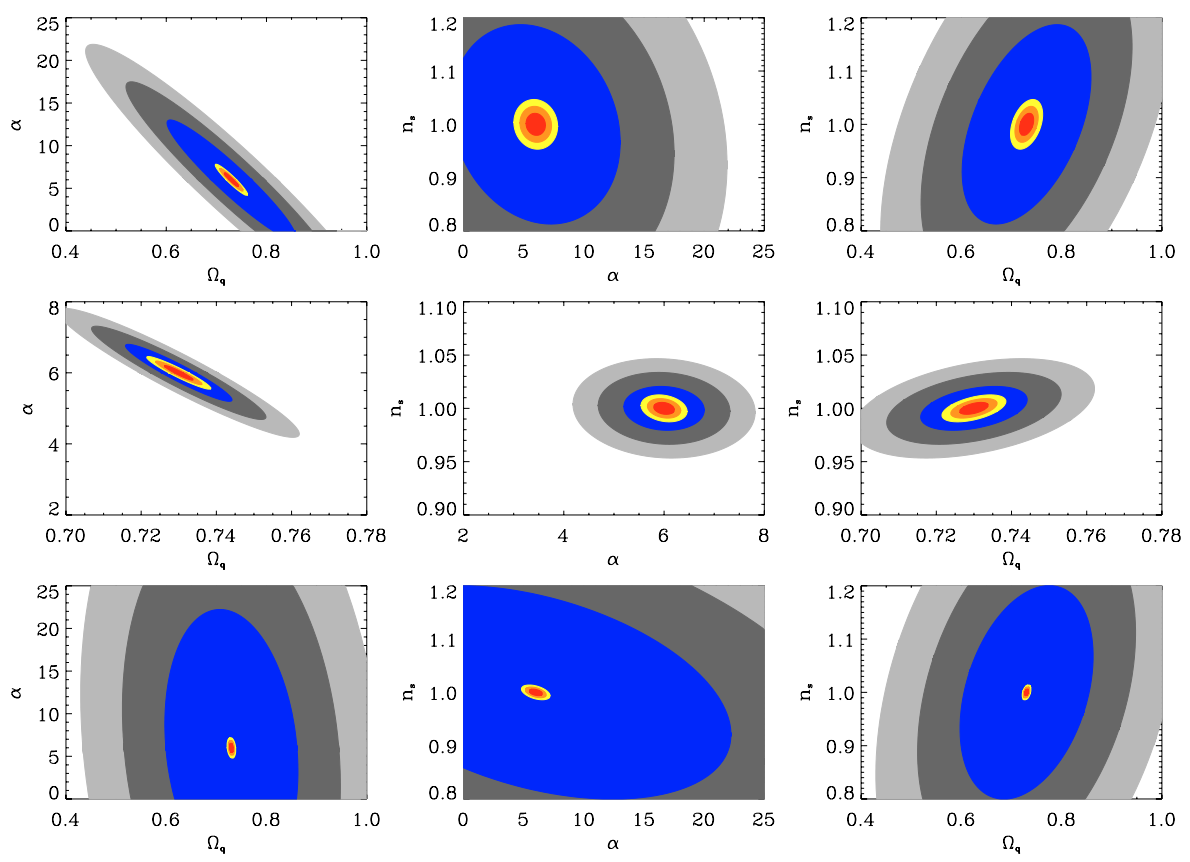

Fig. 13. Fisher analysis of weak lensing (tophat variance) for Ratra-Peebles models, considering a CFHTLS-wide like survey and two space-based survey layouts $(68 \%, 95 \%$, and $99 \%$ C.L.). Upper panels: comparison between a CFHTLS-wide like survey (wider ellipses) and a type $I$ deep space-based survey (inner ellipses) with $A=1000 \mathrm{deg}^{2}, n_{\text {gal }}=$ $50 \mathrm{gal} / \mathrm{arcmin}^{2}, \sigma_{\mathrm{e}}=0.3$. Middle panels: comparison between the type I space-based survey with a wider but shallow space-based survey (type II), with $A=20000 \mathrm{deg}^{2}, n_{\mathrm{gal}}=$ $35 \mathrm{gal} / \mathrm{arcmin}^{2}, \sigma_{\mathrm{e}}=0.3$. Bottom panels: comparison between the CFHTLS-wide like survey with the type II space-based survey, but leaving to vary and then marginalizing over $\tau_{\text {reion }}$. See Sect. 5 for discussion. dealing with high-redshift data and combining them with lowredshift ones. Furthermore, it is complementary to those based on a parameterization of the dark energy sector (Sect. 2), in particular when trying to infer constraints on a physical model from those on the parameters of the equation of state. This also enables one to get rid of the pivot redshift problem when combining different data sets. From a more theoretical point of view, such models cannot lead to an equation of state $w<-1$, contrary to an arbitrary parameterization. It is thus interesting to determine whether there is a tension in the data when such a physical constraint is imposed, as would be concluded from various studies indicating that $w<-1$ is favoured.

To achieve this task, we have used a set of numerical tools that allow us to compute background, CMB and lensing signatures of a large class of cosmological models including quintessence and some extensions such as scalar-tensor theories. We have focused our analysis on three cosmological parameters, the index of the primordial power spectrum, the slope of the quintessence potential, and the quintessence energy density parameter, along with an extra-parameter for the redshift distribution of sources (see Sect. 3.4). This choice was driven by computational limitations, but our analysis will be extended to a larger set of parameters in the near future. Although one can criticise such a small parameter space, it is sufficient to give an idea of the parameter space available for quintessence models and to discuss how weak-lensing data can improve the constraint on dark energy.

We have normalized the initial power spectrum to the CMB so that $\sigma_{8}$ is now a prediction of the models; this parameter is not used for normalization but to check the consistency of results. To account for the non-linear regime of structures formation, on which weak lensing strongly depends when driven by low-redshift galaxies, we have exploited two mappings and discussed their effects on the constraints. In particular, we have shown that, while the determination of the primordial spectrum is sensitive to the chosen mapping, the parameters of the dark energy sector remain robust. Using a synthetic realization of the whole CFHTLS-wide survey, we have also tested the possibility of restricting the analysis using only the large angular scales after cutting the small ones, in order to reduce the influence of the non-linear regime. This analysis confirms the result of the previous one based on real data, showing that the cosmological parameters describing the quintessence field are affected by the non-linear regime to a lesser extent.

We performed a joint analysis including the "goldset" of Sn Ia data and using CMB temperature anisotropies data of the WMAP-1yr release only to normalize the spectra and exclude a region of the quintessence parameter space, based on the location of the first acoustic peak. In particular, our results are not affected by using WMAP-3yr instead of WMAP-1yr data. In contrast, a joint analysis requires a careful calibration of datasets and good control of systematics. The constraints on the two classes of quintessence models considered in this article are shown in Fig. 10 and Table 1. They can be summarised as follows. For a flat universe and a quintessence inverse power-law potential with slope $\alpha$, we get $\alpha<1$ and $\Omega_{\mathrm{Q} 0}=0.75_{-0.04}^{+0.03}$ at a $95 \%$ confidence level, whereas $\alpha=2_{-2}^{+18}, \Omega_{\mathrm{Q} 0}=0.74_{-0.05}^{+0.03}$ when including supergravity corrections.

Finally, we forecast how space-based wide-field imagers will improve our knowledge of dark energy. In particular, we considered two possible strategies, the first deeper and the second wider but shallower. The latter turns out to be more appropriate to track dark energy as far as cosmic shear is concerned.

In the future, we plan to improve this analysis by first comparing it to a similar analysis based on a parameterization of the equation of state, by addressing the problem of the redshift distribution of galaxies more carefully, and by enlarging the parameter space. In particular, for the latter pursuit we would integrate our pipeline with a Markov chain Monte Carlo code (Tereno et al. 2005) developed for the likelihood analysis of cosmic shear. Furthermore, we will be able to also include the analysis of nucleosynthesis constraints, by means of a suitable code for quintessence models (Coc et al. 2006) that is able to deal with ordinary and extended quintessence models like the Boltzmann code and the lensing code used for this study.

Acknowledgements. We thank Nabila Aghanim, Karim Benabed, Francis Bernardeau, Daniel Eisenstein, Bernard Fort, Simon Prunet, Alexandre Réfrégier, and Jim Rich for fruitful discussions. C.S. acknowledges "Fondazione Della Riccia" and "Fondazione Gini" for financial support. IT is partly funded by the CNRS/ANR research grant "ECOSSTAT", contract 
number ANR-05-BLAN-0283-044. LF thanks the "European Association for Research in Astronomy" training site (EARA) and the European Community for the Marie Curie doctoral fellowship MEST-CT-2004-504604. L.V.W. and H.H. are supported by the Natural Sciences and Engineering Research Council (NSERC), the Canadian Institute for Advanced Research (CIAR), and the Canadian Foundation for Innovation (CFI). We thank the CNRS-INSU and the French Programme National de Cosmologie for their support of the CFHTLS cosmic shear program.

\section{References}

Amendola, L. 2000, Phys. Rev. D, 62, 043511

Astier, P., Guy, J., Regnault, N., et al. 2006, A\&A, 447, 31

Athreya, R., Mellier, Y., van Waerbeke, L., et al. 2002, A\&A, 384, 743

Baccigalupi, C., et al. 2002, Phys. Rev. D, 65, 063520

Bacon, D., Réfrégier, A., \& Ellis, R. 2000, MNRAS, 318, 625

Bartelmann, M., \& Schneider, P. 2001, Phys. Rep., 340, 291

Benabed, K., \& Bernadeau, F. 2001, Phys. Rev. D, 64, 083501

Benabed, K., \& van Waerbeke, L. 2003, Phys. Rev. D, 70, 123515

Basset, B. A., Kunz, M., Silk, J., \& Ungarelli, C. 2002, MNRAS, 336, 1217

Basset, B. A., Corasaniti, P. S., \& Kunz, M. 2004, Phys. Rev. D, 69, 083517

Bennett, C. L., Halpern, M., Hinshaw, G., et al. 2003, ApJS, 148, 1

Brax, P., \& Martin, J. 1999, Phys. Lett. B, 468, 40

Brax, P., Martin J., \& Riazuelo, A. 2000, Phys. Rev. D, 62, 103505

Caldwell, R. R., \& Linder, E. V. 2005, Phys. Rev. Lett., 95, 141301

Caldwell, R. R., Dave, R., \& Steinhardt, P. J. 1998, Phys. Rev. Lett., 80, 1582

Caresia, P., Matarrese, S., \& Moscardini, L. 2004, ApJ, 605, 21

Carroll, S. M. 2001, Living Rev. Rel., 4, 1

Chevallier, M., \& Polarski, D. 2001, Int. J. Mod. Phys. D, 10, 213

Chiba, T. 1999, Phys. Rev. D, 60, 083508

Coc, A., et al. 2006, Phys. Rev. D73, 083525

Contaldi, C., Hoekstra, H., \& Lewis, A. 2003, Phys. Rev. Lett., 90, 221

Copeland, E. J., Sami, M., \& Tsujikawa, S. 2006

[arXiv:hep-th/0603057]

Corasaniti, P. S., \& Copeland, E. J. 2003, Phys. Rev. D, 67, 063521

Corasaniti, P. S., et al. 2004, Phys. Rev. D, 70, 083006

Crocce, M., \& Scoccimarro, R. 2005, Phys. Rev. D73, 063519; Phys. Rev. D73, 063520

Dave, R., Caldwell, R. R., \& Steinhardt, P. J. 2002, Phys. Rev. D, 66, 023516

Dolag, K., et al. 2003, A\&A, 416, 853

Doran, M., Schwindt, J., \& Wetterich, C. 2001a, Phys. Rev. D, 64, 123520

Doran, M., Lilley, M., Schwindt, J., \& Wetterich, C. 2001b, ApJ, 559, 501

Doran, M., \& Lilley, M. 2002, MNRAS, 330, 965

Eisenstein, D. J., Zehavi, I., Hogg, D. W., et al. 2005, ApJ, 633, 560

Hamilton, A. J., Kumar, P., Lu, E., Matthews, A., et al. 1991, ApJ, 374, L1

Hinshaw, G., Spergel, D. N., Verde, L., et al. 2003, ApJS, 148, 135

Hinshaw, G., Nolta, M. R., Bennett, C. L., et al. 2006 [arXiv: astro-ph/0603451]

Hoekstra, H. 2003, IAU Symp. 216, Maps of the Cosmos, ASP Conf. Ser., ed. M. Colless, \& L. Staveley-Smith, Sydney [arXiv:astro-ph/0310908]

Hoekstra, H., Mellier, Y., van Waerbeke, L., et al. 2006, ApJ647, 116

Hu, W., \& Jain, B. 2004, Phys. Rev. D, 70, 043009

Hu, W., \& White, M. 1997, Phys. Rev. D, 56, 596

Huterer, D., \& Takada, M. 2005, Astropart. Phys., 23, 369

Ishak, M., Hirata, C., Mc Donald, P., \& Seljak, U. 2004, Phys. Rev. D, 69, 083514

Jain, B., \& Taylor, A. 2003, Phys. Rev. Lett., 91, 141302

Jarosik, N., Barnes, C., Greason, M. R., et al. 2006 [arXiv: astro-ph/0603452]

Jarvis, M., Jain, B., Bernstein, G., \& Dolney, D. 2006, ApJ, 644, 71

Jassal, H. K., Bagla, J. S., \& Padmanabhan, T. 2004, MNRAS, 356, L11

Jing, Y. P., Zhang, P., Lin, W. P., Gao, L., \& Springel, V. 2005

[arXiv:astro-ph/0512426]
Kaiser, N., Wilson, G., \& Luppino, G. 2000 [arXiv: astro-ph/0003338] Kamionkowski, M., \& Buchalter, A. 2000 [arXiv : astro-ph/0001045]

Klypin, A., Macciò, A. V., Mainini, R., \& Bonometto, S. A. 2003, ApJ, 599, 31

Kujat, J., Linn, A. M., Scherrer, R. J., \& Weinberg, D. H. 2002, ApJ, 572, 1

Linder, E. V. 2003, Phys. Rev. Lett., 90, 091301

Linder, E. V., \& Huterer, D., 2005, Phys. Rev. D, 72, 043509

Ma, C. P., \& Fry, J. N. 2000, ApJ, 543, 503

Ma, C. P., Caldwell, R. R., Bode, P., \& Wang, L. 1999, ApJ, 521, L1

Mainini, A., Macciò, A. V., Bonometto, S. A., \& Klypin, A. 2003, ApJ, 599, 24

Martin, J., Schimd, C., \& Uzan, J.-P., 2006, Phys. Rev. Lett., 96, 061303

McCracken, H. J., Radovich, M., Bertin, E., et al. 2003, A\&A, 410, 17

McDonald, P., Trac, H., \& Contaldi, C. 2006, MNRAS, 366, 547

Mellier, Y. 1999, ARA\&A, 37, 127

Padmanabhan, T. 2003, Phys. Rep., 380, 235

Page, L., Nolta, M. R., Barnes, C., et al. 2003, ApJS, 148, 233

Page, L., Hinshaw, G., Komatsu, E., et al. 2006 [arXiv: astro-ph/0603450]

Peacock J. A., \& Dodds, S. J. 1996, MNRAS, 280, L19

Peebles, P. J. E. 1993, Principles of physical cosmology (Princeton University Press)

Peebles P. J. E., \& Ratra, B. 2003, Rev. Mod. Phys. 75, 559.

Peter, P., \& Uzan, J.-P. 2005, Cosmologie primordiale (Belin)

Podariu, S., \& Ratra, B. 2000, ApJ, 532, 109

Ratra, B., \& Peebles, P. J. E. 1988, Phys. Rev. D, 37, 3406

Refregier, A. 2003, ARA\&A, 41, 645

Riazuelo, A., \& Uzan, J.-P. 2000, Phys. Rev. D, 62, 083506

Riazuelo, A., \& Uzan, J.-P. 2002, Phys. Rev. D, 66, 023525

Riess, A. G., Strolger, L.-G., Tonry, J., et al. 2004, ApJ, 607, 665

Sachs, R. K. 1962, Proc. Roy. Soc. A, 270, 103

Sandvik, H. B., et al. 2004, Phys. Rev. D, 69, 063005

Scherrer, R. J. 2005, Phys. Rev. D, 73, 043502

Schimd, C., Uzan, J.-P., \& Riazuelo, A. 2005, Phys. Rev. D, 71, 083512

Schneider, P., Ehlers, J., \& Falco, E. E. 1992, Gravitational lenses (Springer)

Schneider, P., van Waerbeke, L., Kilbinger, M., \& Mellier, Y. 2002, A\&A, 396,

Seljak, U. 2000, MNRAS, 318, 203

Semboloni, E., Mellier, Y., van Waerbeke, L., et al. 2006, A\&A, 452, 51

Simpson, F., \& Bridle, S. 2005, Phys. Rev. D, 71, 083501

Smith, R. E., Peacock, J. A., Jenkins, A., et al. 2003, MNRAS, 341, 1311

Spergel, D. N., et al. 2003, ApJS, 594, 1

Spergel, D. N., Bean, R., Dore', O., et al. 2006 [arXiv: astro-ph/0603449]

Stebbins, A. 1996 [arXiv: astro-ph/9609149]

Tereno, I., Doré, O., van Waerbeke, L., \& Mellier, Y. 2005, A\&A, 429, 383

Uzan, J.-P. 1999, Phys. Rev. D, 59, 123510

Uzan, J.-P. 2004, AIP Conf. Proc. 736, Phi in the Sky: The Quest of Cosmological Scalar Fields [arXiv: astro-ph/0409424]

Uzan, J.-P., \& Bernardeau, F. 2000, Phys. Rev. D, 63, 023004

Uzan, J.-P., \& Bernardeau, F. 2001, Phys. Rev. D, 64, 083004

Uzan, J.-P., Aghanim, N., \& Mellier, Y. 2004, Phys. Rev. D, 70, 083533

van Waerbeke, L., \& Mellier, Y. 2003, Lecture given at the Aussois winter school, France [arXiv: astro-ph/0305089]

van Waerbeke, L., Mellier, Y., Erben, T., et al. 2000, A\&A, 358, 30

van Waerbeke, L., Mellier, Y., Radovich, M., et al. 2001, A\&A, 374, 757

van Waerbeke, L., Mellier, Y., \& Hoekstra, H. 2005, A\&A, 429, 75

van Waerbeke, L., White, M., Hoekstra, H., \& Heymans, C. 2006, Astropart. Phys. 26, 91

Verde, L., Peiris, H. V., Spergel, D. N., et al. 2003, ApJS, 148, 195

Wetterich, C. 1988, Nucl. Phys. B, 302, 668

White, M. 2004, Astropart. Phys., 22, 221

White, M., \& Vale, C. 2004, Astropart. Phys., 22, 19

Wittman, D. M., Tyson, J. A., Kirkman, D., Dell'Antonio, I., \& Bernstein, G. 2000, Nature, 405, 143

Yahata, N., Lanzetta, K. M., \& Chen, H.-W. 2000, ApJ, 538, 493

Zhan, H., \& Knox, L. 2004, ApJ, 616, L75

Zlatev, I., Wang, L., \& Steinhardt, P. J. 1999, Phys. Rev. Lett., 82, 896 